\title{
Modeling of contact stress and tool-based frictional forces considering edge effect through material separation exploration
}

\section{Weiwei Zhang}

Wuhan University of Technology

Jian Weng

Wuhan University of Technology

Kejia Zhuang ( $\sim$ zhuangkj@whut.edu.cn )

Wuhan University of Technology

Cheng Hu

Wuhan University of Technology

Xing Dai

Wuhan University of Technology

Chaoqun Wu

Wuhan University of Technology

\section{Research Article}

Keywords: Tool-based cutting forces, Contact behavior, Separation point, Dead metal zone, Rounded edge tool

Posted Date: March 15th, 2021

DOI: https://doi.org/10.21203/rs.3.rs-313988/v1

License: (9) This work is licensed under a Creative Commons Attribution 4.0 International License.

Read Full License 


\title{
Modeling of contact stress and tool-based frictional forces considering edge effect through material separation exploration
}

\author{
Weiwei Zhang ${ }^{1}$, Jian Weng ${ }^{1}$, Kejia Zhuang ${ }^{1}{ }^{*}$, Cheng $\mathrm{Hu}{ }^{1}$, Xing Dai ${ }^{2}$, Chaoqun $\mathrm{Wu}^{1}$ \\ ${ }^{1}$ Hubei Digital Manufacturing Key Laboratory, School of Mechanical and Electronic Engineering, Wuhan \\ University of Technology, Wuhan 430070, China \\ ${ }^{2}$ State Key Laboratory of Digital Manufacturing Equipment and Technology, Huazhong University of \\ Science and Technology, Wuhan, China
}

\begin{abstract}
Cutting tools with round edge can enhance the performance of machining difficult-tomachine materials, while the complex contact mechanism related to micro cutting edge limits the deeper understanding of cutting mechanics. Material separation, which is associate to plough mechanism with formation of dead metal zone (DMZ), also requires the analysis of contact behavior This study develops a contact model along the round edge together with the illustration of DMZ, with three contact feature points defined to explain the contact situation between workpiece and cutting edge. Among these feature points, two separation points related to DMZ classify the sliding and sticking region considering the dual-zone approach. The stagnation point is the zero shear stress point where a sudden change in shear stress direction happens. Besides, the parabolic stress model obtained from finite element simulations is established to define the normal contact distribution along the round edge. In this basis, the tool-based frictional forces are determined and two contact force components are classified for different contact regions. The proposed contact feature points and contact stress are validated through illustration with finite element simulations. Besides, orthogonal cutting tests ensure the practicality and accuracy of the proposed contact model and predicted cutting forces.
\end{abstract}

Keywords: Tool-based cutting forces, Contact behavior, Separation point, Dead metal zone, Rounded edge tool

\footnotetext{
* Corresponding author: Kejia Zhuang; Email address: zhuangkj@whut.edu.cn
} 


\section{Introduction}

Machining difficult-to-machine materials is the focus of research in manufacturing and the mechanical and chemical properties of these materials cause high wear on cutting edges [1-3]. The mechanical strength of cutting tool is enhanced by preparing cutting edges with specific rounding. In the processes with rounded edge tool, the phenomenon that the selected uncut chip thickness is always the same order magnitude of edge radius cannot be ignored. The plough mechanism resulted from round edge leads to the fact that part of material is pressed into workpiece [4,5]. The traditional cutting force model described the plough mechanism as edge force component and thought this component is independent with uncut chip thickness. However, uncut chip thickness affects the plough mechanism and this mechanism needs to explore in detail in cutting, especially in machining difficult-to-machine materials

Many researchers studied cutting forces based on the plough mechanism exploration and cutting processes. Albrecht [6] first presented plough mechanism based on the fact that the cutting edge is not completely sharp. Then, the slip line theory is popularly used by many researchers to explain plough mechanism in cutting force modeling [7-10]. The establishment of slip line field mainly focuses on the identification of the DMZ geometrical structure, which exists in the front of rake face and keeps the same speed as cutting tool. In recent years, finite element analysis is used in slip line modeling for its cheapness and efficiency. Hu et al. [11] used the orthogonal cutting model through the Advantedge software to modify the slip line theory. Jin and Altintas [12] compared micro-milling forces achieved by finite element simulation and slip line modeling. Some researchers used finite element simulation to obtain plough force directly. In milling with Brass 260 , Jin et al. [12] predicted plough forces through a 2D finite element model covered by an arbitrary Lagrangian-Eulerian way. A 3D turning simulation model is established by Zhou et al. [13] to predict three-dimensional turning force components affected by both edge radius and nose radius.

Some researchers found that there was a deviation of feed force achieved between simulation and experiment due to irrational selection of contact friction [12, 14, 15]. Sampsa et al. [16] explored that the friction selection affected the feed force component by $20-50 \%$. In fact, the exploration aiming at the contact behavior between the round edge and workpiece is a key point in cutting forces research. Woon et al. [17] determined the friction distribution along the round edge based on DMZ with cutting hardened steel and the contact stress reached maximum around the stagnation point. Ulutan and Özel [1] found that excessive tool wear occurred in machining nickel-based alloys and the normal contact stress kept constant along the round edge. Wang et al. [18] researched the cutting forces in micro milling of difficult-to-cut material based on contact situation exploration. In their model, the normal contact stress along the round edge was expressed as a parabolic equation and the maximum normal contact stress occurred at the end of clearance roundness. 

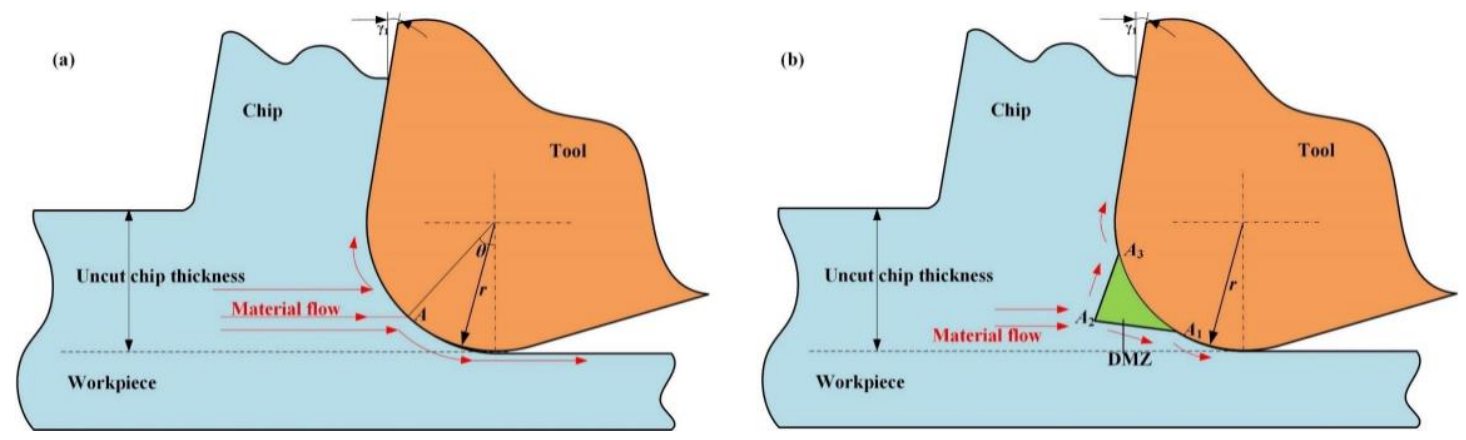

Fig. 1 The classical material separation theory

(a. Material separation proposed by Basuray et al. [20], b. Material separation based on DMZ

All contact stress models above only focused on the stagnation point or ignored the material separation resulted from round edge. In fact, exploring material separation is important in modeling contact situation. As shown in Fig. 1, there are two different representative theories about material separation with round edge. Basuray et al. [19] thought there was a stagnation point (point A) illustrated in Fig. 1(a), the material above point A formed chip and that below this point flowed into the machined surface. The existence of this point was also verified through the explicit analytical expression established by Yuan et al. [20]. In recent years, some advanced technologies, including finite element analysis [21] and acoustic emission signals [22], are gradually used to identify the position of the separation point. As shown in Fig. 1(b), Waldorf et al. [7] held a belief that the material separation happened at the extreme edge of the build-up in slip line modeling. Material separation is decided through dead metal zone assumption and there are three separation points $\left(A_{1}\right.$, $A_{2}$ and $\left.A_{3}\right)$. Wan et al. [23] thought the shear strain associated with $A_{1}$ and $A_{3}$ is infinite and the infinite shear strain theory was used to predict the positions of these two separation points.

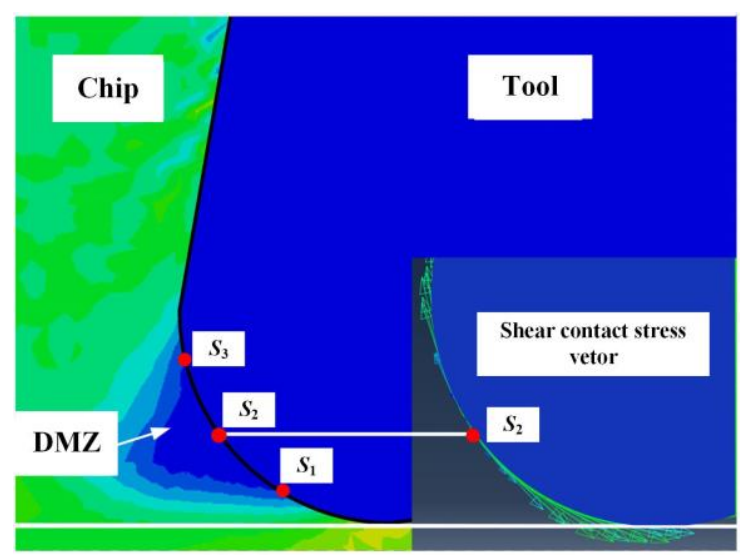

Fig. 2 Tool-workpiece contact modeling considering round edge

Differ from the previous studies only focusing on material flow, this study describes material separation from the perspective of friction situation. As shown in Fig. 2, this research finds there are three contact feature points along the round edge contact region, i.e. $S_{1}, S_{2}$, and $S_{3}$ through finite element simulation. It is thought that sticking friction condition occurs between DMZ and cutting tool. $S_{1}$ and $S_{3}$ are separation points associated with DMZ and these two points limit the size of DMZ. $S_{2}$ is defined through shear contact stress veto analysis along the round edge. The shear contact stress related to $S_{2}$ is zero and opposite direction of shear contact stress occurs above and 
below $S_{2}$. These defined contact points are used to set up the contact model with round edge.

This paper mainly researches the cutting forces modeling with contact definition affected by round edge and then, a new contact stress model is proposed based on the contact feature point definition. In this proposed model, normal contact stress along the round edge is defined through the parabolic model. The shape of DMZ is used to determine the sticking region and sliding region. Then, tool-based cutting forces are predicted with contact model parameters and these parameters are identified properly through iterative solution methodology with experimental data. As a result, the contact stress distribution along both rake face and round edge are identified clearly. Besides, the effect of edge radius on tool-workpiece contact distribution is explored in detail. More importantly, two different contact force components are extracted to research the effect of edge radius on total cutting forces.

There are six sections in this paper. In section 1, the background of cutting forces considering round edge and the novelty of this work are introduced. Section 2 gives the contact model and toolbased force model in detail. The optimization algorithm searching the model variables is discussed in section 3. In section 4, numerical simulation tests and orthogonal cutting experiments are illustrated to validate the proposed model. The research results including contact stress and cutting force components are discussed in section 5 . The conclusions are drawn in section 6 .

\section{Cutting forces prediction considering contact behavior}

\subsection{Contact behavior modeling}

This study focuses on the contact situation in cutting with rounded edge tool. Some points are proposed to cover the contact situation shown in Fig. 3. $\gamma_{1}$ is rake angle, $\gamma_{2}$ is clearance angle and edge radius is expressed as $r . S_{0}$ is the end of tool-work contact and $S_{4}$ is the end of rake roundness. $S_{1}-S_{3}$ are contact feature points defined in section 1 and $S_{5}$ is the end of tool-chip contact. Besides, $l$ represents the distance along the rake face from $S_{4}$ and $\alpha$ is the angle measured from feed direction of round edge.

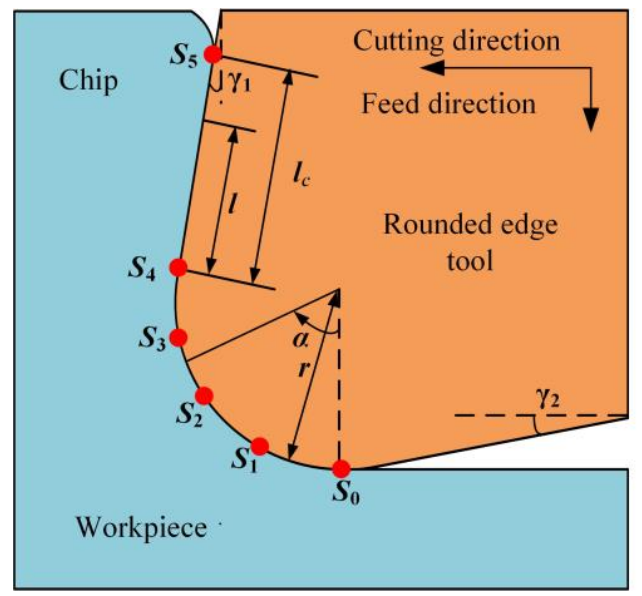

Fig. 3 Contact stress points defined with round edge

In order to define the contact stress more systematically, this study divides the whole contact region into two different contact regions, including round edge contact region $\left(S_{0}-S_{4}\right)$ and rake face contact region $\left(S_{4}-S_{5}\right)$. The round edge region is defined as the contact area between workpiece and round edge. The orthogonal cutting simulations based on ABAQUS are illustrated to research the contact behavior along the round edge. The materials of work and tool are Ti-6Al-4V and carbide, 
respectively. In simulation tests, the edge radius ranges from $0.01 \mathrm{~mm}$ to $0.05 \mathrm{~mm}$ and the full interaction between work and round edge are ensured. Fig. 4 shows the normal contact stress along the round edge through finite element simulation with $r=0.05 \mathrm{~mm}$. Clearly, the normal contact stress changes nonlinearly of $\alpha$. Specifically, with $\alpha$ changing from $0^{\circ}$ to $\alpha_{4}$ (expressed in Eq. (1)), normal contact stress increases firstly and reaches the maximum value around $S_{2}$, then it falls nonlinearly. Thus, the normal contact stress related to $S_{2}$ is expressed in Eq. (2). $\sigma\left(\alpha_{2}\right)$ is the normal contact stress of point $S_{2}$. It's found that the simulated values can be fitted with a parabolic curve. Thus, this study assumed the relationship between normal stress and $\alpha$ to be the parabolic function. The selected parabolic equation is expressed in Eq. (3). $\sigma(\alpha)$ is the normal contact stress along the round edge and $c_{k}$ are model parameters.

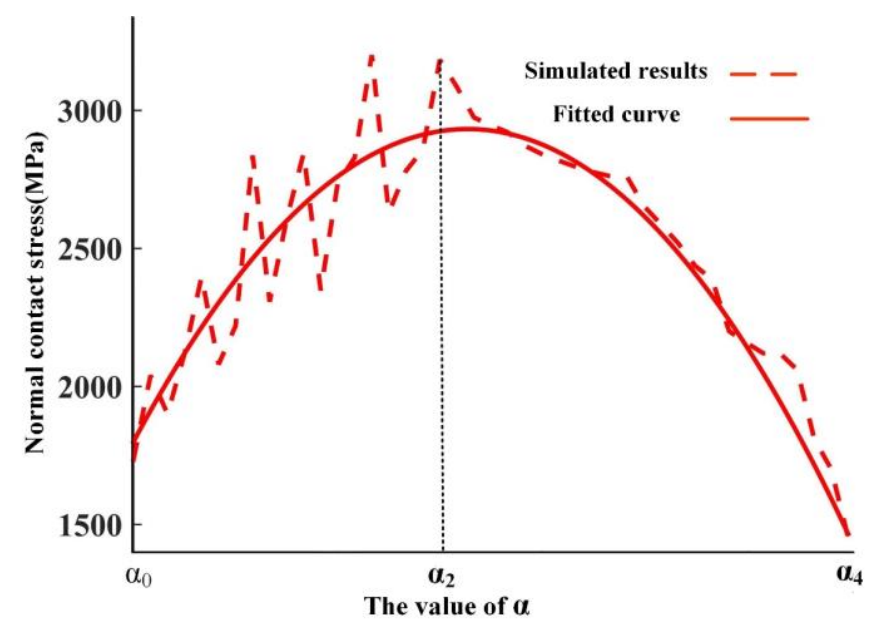

Fig. 4 Normal contact stress modeling through simulation and regression

$$
\begin{gathered}
\alpha_{4}=\gamma_{1}+\frac{\pi}{2} \\
\frac{d \sigma\left(\alpha_{2}\right)}{d \alpha}=0 \\
\sigma(\alpha)=\sum_{k=0}^{2} c_{k} \alpha^{k}
\end{gathered}
$$

As for the shear contact stress, the sticking region and sliding region are distinguished clearly with dead metal zone. In the contact region of round edge, DMZ exists in the front of round edge. Some researchers regarded the boundaries of dead metal zone as the boundaries of the plastic deformation zone [7]. Considering the dual-zone approach used in contact definition, it is believed that sticking contact occurs in plastic deformation zone [24]. A finite element cutting simulation aiming at DMZ is accomplished in Fig. 2 and clearly, $S_{1}$ and $S_{3}$ are two points that define the plastic deformation boundaries. Therefore, sticking contact happens in region $S_{1} S_{3}$, while sliding contact happens in region $S_{0} S_{1}$ and region $S_{3} S_{4}$. In the sliding contact region, the friction contact coefficient is constant and there is the limiting shear stress in sticking region. Besides, the shear contact stress directions in the contact region above $S_{2}$ are opposite to these below $S_{2}$ and the shear contact stress related to $S_{2}$ is zero. The direction of shear contact vector along contact region above the $S_{2}$ is set positive and $\alpha$ is used to express the location of contact feature points. As a result, the shear contact stress in the round edge contact region is expressed in Eq. (4). $\tau_{\max }$ is the limiting shear stress and $\tau(\alpha)$ shear contact stress along the round edge. 


$$
\left\{\begin{array}{cc}
\tau(\alpha)=-\mu \sigma(\alpha) & 0 \leq \alpha<\alpha_{1} \\
\tau(\alpha)=-\tau_{\max } & \alpha_{1} \leq \alpha<\alpha_{2} \\
\tau(\alpha)=0 & \alpha=\alpha_{2} \\
\tau(\alpha)=\tau_{\max } & \alpha_{2}<\alpha \leq \alpha_{3} \\
\tau(\alpha)=\mu \sigma(\alpha) & \alpha_{3}<\alpha \leq \alpha_{4}
\end{array}\right.
$$

The distribution of contact stress along the round edge is identified based on DMZ. Thus, determining the location of DMZ is extremely important. Three points $\left(S_{1}, S_{3}, S_{6}\right)$ are used to define the geometrical model of this zone shown in Fig. 5(a). It is found that materials slide out and separate due to shearing effect at the $S_{1}$ and $S_{3}$.

(a)

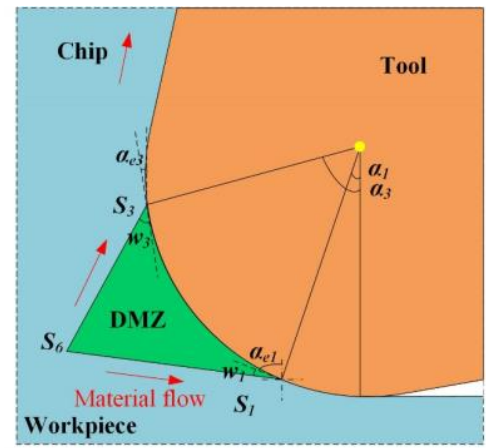

(b)

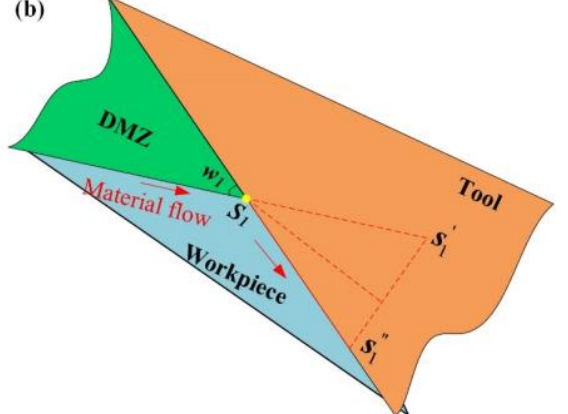

Fig. 5 Shearing effect during the formation of DMZ

(a. The geometrical model of DMZ; b. The strain situation along the point $\mathrm{S}_{1}$ )

The infinite shear strain theory is used to explain the shear effect as expressed in Eq. (5) [23]. $\lambda_{1}$ and $\lambda_{3}$ are shear strain of workpiece material related to $S_{1}$ and $S_{3}$, respectively. Taking the strain situation related to point $S_{1}$ as an example shown in Fig. 5(b), the materials flow along the $S_{1} S_{6}$ and then reach $S_{1}^{\prime}$ if there is no round edge. Thus, the shear strain at point $\mathrm{S}_{1}$ is expressed as Eq. (6). $\angle$ $S_{1} S^{\prime}{ }_{1} S_{1}{ }_{1}$ is shear angle and Eq. (7) is obtained based on the maximum shear stress principle. $\beta$ and $\alpha_{e l}$ are friction angle and effective rake angle related to point $S_{1}$, respectively. Besides, in $\Delta S_{1} S^{\prime}{ }_{1} S^{\prime \prime}{ }_{1}$, $\angle S_{1} S^{\prime \prime}{ }_{1} S_{1}^{\prime}$ is achieved according to Eq. (8). $w_{1}$ is the angle between the slip line and plastic area boundary[25], which associates with the friction factor as expressed as Eq. (9). $\mu$ is the friction coefficient and is related to $\beta$ as expressed in Eq. (10). Besides, in round edge region, the relationship between effective rake angle and $\alpha$ is displayed in Eq. (11). From Eq. (5) to Eq. (11), the values of $\alpha$ related to $S_{1}$ and $S_{3}$ are obtained.

$$
\begin{gathered}
\lambda_{1}=\lambda_{3}=0 \\
\lambda_{1}=\cot \angle s_{1} s_{1}^{\prime} s_{1}^{\prime \prime}+\cot \angle s_{1} s_{1}^{\prime \prime} s_{1}^{\prime} \\
\angle s_{1} s_{1}^{\prime} s_{1}^{\prime \prime}=\frac{\pi}{4}-\left(\beta-\alpha_{e 1}\right) \\
\angle s_{1} s_{1}^{\prime \prime} s_{1}^{\prime}=\pi-\angle s_{1} s_{1}^{\prime} s_{1}^{\prime \prime}-w_{1} \\
\left(\sqrt{3}+\sin 2 w_{1}\right) \mu=\cos 2 w_{1} \\
\mu=\tan \beta
\end{gathered}
$$




$$
\alpha_{e 1}=-\left(\frac{\pi}{2}-\alpha_{i}\right)
$$

In the contact region of rake face $\left(S_{4}\right.$ to $\left.S_{5}\right), l$ varies from zero to $l_{c}$. The ratio of contact length $\left(l_{c}\right)$ to uncut chip thickness $(h)$ is expressed as $l_{c a}$, which is shown in Eq. (12). The normal contact stress $(\sigma(l))$ is defined as a function that varies with $l$ expressed in Eq. (13) [1]. Normal contact stress decreases nonlinearly with $l$ increasing and $\sigma_{\max }$ is the normal contact stress related to $S_{4}$. As for shear contact stress along the rake face, the sticking region has been limited with point $S_{2}$ and $S_{3}$ for the existence of dead metal zone. The material separates in $S_{3}$, and then slides along the rake face to form the machined chip. Thus, it is thought that the rake face region belongs to the sliding region and the shear contact stress $(\tau(l))$ is achieved through Eq. (14).

$$
\begin{gathered}
l_{c a}=\frac{l_{c}}{h} \\
\sigma(l)=\sigma_{\max }\left(1-\frac{l}{l_{c}}\right)^{n_{c}} \\
\tau(l)=\mu \sigma(l)
\end{gathered}
$$

\subsection{Cutting forces prediction with stresses distribution}

In this study, cutting forces can be determined based on contact behavior which gives a detailed information of the contact stresses. In Fig. 6, $F_{t c}$ and $F_{f c}$ are force components of rake face in cutting and feed direction, respectively. $F_{t r}$ and $F_{f r}$ are force components of round edge in cutting and feed direction, respectively. The total cutting forces are expressed in Eq. (15). $F_{t}$ and $F_{f}$ are total forces in cutting and feed direction, respectively.

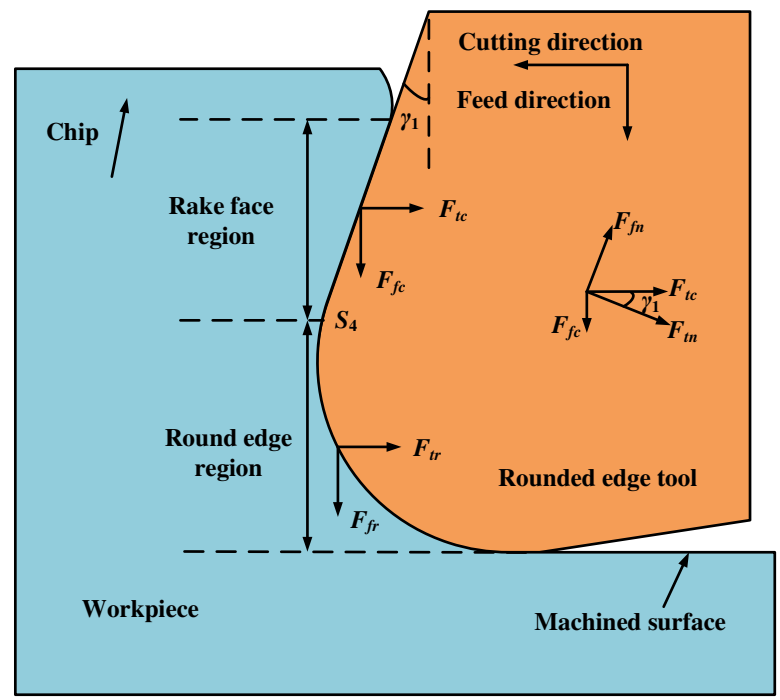

Fig.6 Force components in cutting with rounded edge tool

$$
\left\{\begin{array}{l}
F_{t}=F_{t c}+F_{t r} \\
F_{f}=F_{f c}+F_{f r}
\end{array}\right.
$$

In contact region of rake face, the normal contact stress and shear contact stress are expressed in Eq. (13) and Eq. (14). Integrating from $S_{4}$ to $S_{5}$, the values of $F_{t c}$ and $F_{f c}$ are achieved expressed in Eq. (16). 


$$
\left\{\begin{array}{l}
F_{t c}=\int_{0}^{l_{c}} b\left(\cos \gamma_{1}+\mu \sin \gamma_{1}\right) \sigma_{l} d l \\
F_{f c}=\int_{0}^{l_{c}} b\left(\sin \gamma_{1}-\mu \cos \gamma_{1}\right) \sigma_{l} d l
\end{array}\right.
$$

Eq. (1) to Eq. (4) show the contact behavior related to contact region in round edge. Integrating all functions among the whole region, the values of $F_{t r}$ and $F_{f r}$ are calculated in Eq. (17).

$$
\left\{\begin{array}{l}
F_{t r}=\int_{\alpha_{0}}^{\alpha_{4}} b r_{e}(\sigma(\alpha) \sin \alpha-\tau(\alpha) \cos \alpha) d \alpha \\
F_{f r}=\int_{\alpha_{0}}^{\alpha_{4}}-b r_{e}(\sigma(\alpha) \cos \alpha+\tau(\alpha) \sin \alpha) d \alpha
\end{array}\right.
$$

\section{The determination of model variables}

Section 2 shows the modeling of tool-based cutting forces in detail and some contact model variables are selected properly through the optimization algorithm in this section. The geometrical constraints include $\gamma_{1}$ and $r$ from rounded edge tool. The contact variables are $l_{c}, \sigma_{\max }, \tau_{\max }$ and contact model parameters of round edge region $\left(c_{0}, c_{1}, c_{2}\right.$, and $\left.\mu\right)$. Among all these variables, $\tau_{\max }$ and $\sigma_{\max }$ is determined through Eq. (18) and Eq. (19), respectively. In the proposed model, the shear stress related to $S_{1}$ is equal to it related to $S_{3}$. As a result, the relationship between $c_{1}$ and $c_{2}$ is achieved in Eq. (20).

$$
\begin{gathered}
\sigma_{\text {max }}=\sigma\left(\alpha_{4}\right) \\
\tau_{\text {max }}=\tau\left(\alpha_{1}\right) \\
c_{1}=\frac{-c_{2}}{\alpha_{1}+\alpha_{3}}
\end{gathered}
$$

As analyzed above, four variables $l_{c a}, c_{0}, c_{2}$, and $\mu$ need to be selected properly. In Fig. 6 , the relationship between frictional forces and rake face force components is also expressed. The force components determined by frictional forces are displayed in Eq. (21), $F_{t n}$ and $F_{t f}$ are the normal and shear contact force components along rake face, respectively. It should be noted that $F_{t f}$ can be calculated through $F_{t n}$ shown in Eq. (22).

$$
\begin{gathered}
\left\{\begin{array}{c}
F_{t c}=F_{t n} \cos \gamma_{1}+F_{t f} \sin \gamma_{1} \\
F_{f c}=F_{t n} \sin \gamma_{1}-F_{t f} \cos \gamma_{1}
\end{array}\right. \\
F_{t f}=\mu F_{t n}
\end{gathered}
$$

In cutting experiments with rounded edge tool, the extraction of cutting force components along the rake face is difficult to accomplish directly. The force increment is explored to determine the frictional coefficient. In the contact model, it is assumed that the contact situation of round edge is independent with uncut chip thickness under the premise that the interaction along the rake face region occurs. In another word, when uncut chip thickness is large enough, the force components of round edge keep constant and the increment of total cutting forces is equal to the increment of rake face force components. Therefore, the relationship between cutting force increments and the frictional coefficient is expressed in Eq. (23). $\Delta F_{t}, \Delta F_{f}$ and $\Delta F_{t n}$ are the increment of the total force 
in cutting direction, total force in feed direction and normal contact force component, respectively. Then, separate $\mu$ to the left of the equation, Eq. (24) is achieved. Finally, the value of $\mu$ is achieved based on experimental cutting forces.

$$
\begin{gathered}
\left\{\begin{array}{l}
\Delta F_{t}=\Delta F_{t n}\left(\cos \gamma_{1}+\mu \sin \gamma_{1}\right) \\
\Delta F_{f}=\Delta F_{t n}\left(\sin \gamma_{1}-\mu \cos \gamma_{1}\right)
\end{array}\right. \\
\mu=\frac{\Delta F_{t} \sin \gamma_{1}+\Delta F_{f} \cos \gamma_{1}}{\Delta F_{t} \cos \gamma_{1}-\Delta F_{f} \sin \gamma_{1}}
\end{gathered}
$$

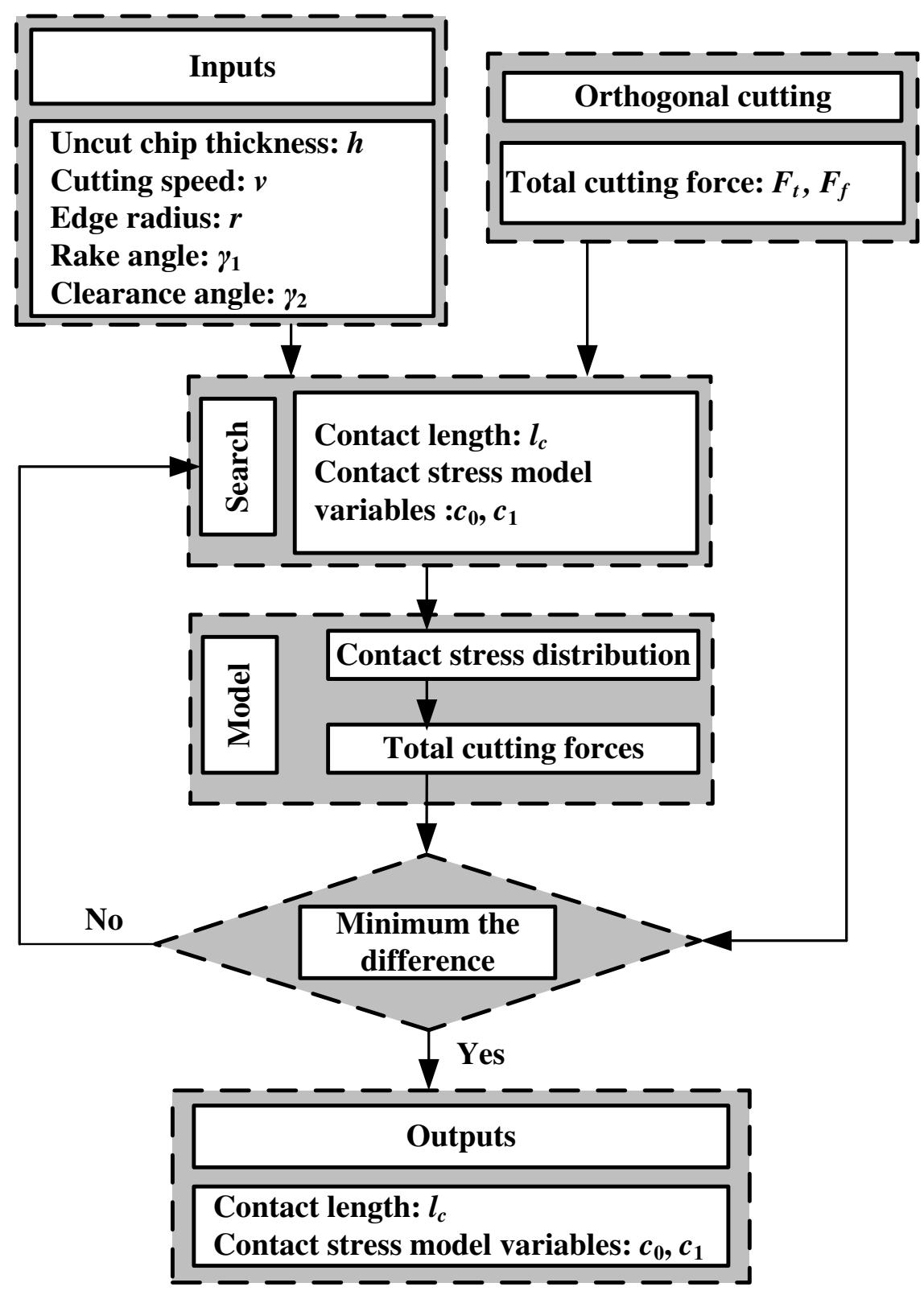

Fig. 7 Illustration of search and optimization algorithm

As for the achievement of $l_{c a}, c_{0}$ and $c_{2}$, the optimization algorithm is used in this research. As illustrated in Fig. 7, these three variables are searched at every iteration [1]. At the first iteration, some meaningful limitations are set in selecting three unknown variables. For example, the value 
of $l_{c a}$ varies between 1 and 2, which is concluded by Fang [8]. These variables are selected at random and the analytical total cutting forces are determined by contact model defined in section 2 . These analytical forces are compared to the experimental forces from cutting experiment, and the search process reaches the end when the error is in the set error range.

\section{Model validation}

The proposed contact feature points and contact stress distribution are calibrated through numerical simulation. The predicted cutting forces are compared with both published results and experimental data. Besides, in order to ensure the accuracy of proposed contact model, the contact length and contact surface are identified through Alicona.

\subsection{Validation of material separation}

In this section, finite element simulations are used to validate material separation in cutting with rounded edge tool. The numerical simulation is established through the software ABAQUS and the geometrical model of $2 \mathrm{D}$ cutting simulation is shown in Fig. 8 . The rake angle is $10^{\circ}$ and the clearance angle is selected as $8^{\circ}$. The edge radius varies from $0.02 \mathrm{~mm}$ to $0.05 \mathrm{~mm}$ and uncut chip thickness changes from $0.1 \mathrm{~mm}$ to $0.20 \mathrm{~mm}$. $v$ is cutting speed, which is selected as $70 \mathrm{~m} / \mathrm{min}$. Besides, the element type of cutting tool and the workpiece is CPE3T and CPE4RT, respectively.

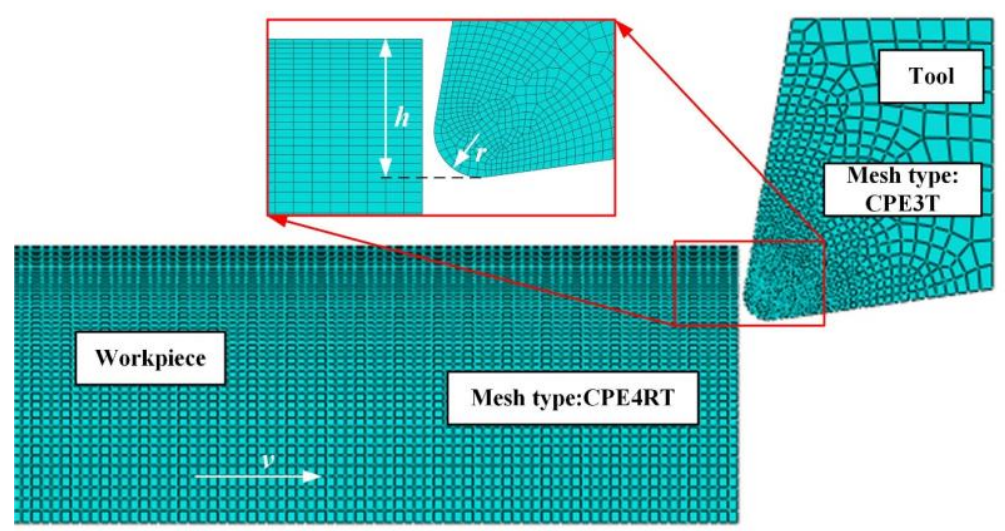

Fig. 8 Geometrical modeling in finite element simulation

Table 1 The material property parameters

\begin{tabular}{|c|c|}
\hline Material & Ti-6Al-4V \\
\hline Density (ton $/ \mathrm{mm}^{3}$ ) & $4.44 \mathrm{e}-9$ \\
\hline Specific heat $\left(\mathrm{mJ} /\right.$ ton $\left.^{\circ}{ }^{\circ} \mathrm{C}\right)$ & $0.58 \mathrm{e} 9$ \\
\hline Coefficient Of Thermal expansion $\left({ }^{\circ} \mathrm{C}^{-1}\right)$ & $9.3 e-6$ \\
\hline Young's Modulus (MPa) & 110000 \\
\hline Thermal conductivity $\left(\mathrm{w} / \mathrm{m} \cdot{ }^{\circ} \mathrm{C}\right)$ & 10.4 \\
\hline Poisson's ratio & 0.34 \\
\hline Room temperature $\left({ }^{\circ} \mathrm{C}\right)$ & 20 \\
\hline
\end{tabular}

As for the physic model, Ti-6Al-4V is workpiece material and the material properties are given in Table 1. The Johnson-Cook (J-C) constitutive model [26] is used to define the work material plastic deformation. As expressed in Eq. (25), the J-C model covered the effect of temperature, strain and strain rate on material deformation comprehensively, thus it has been used widely in cutting 
simulation. In this model, $A, B, C, n$ and $m$ represent the initial yield stress, strength coefficient, strain-rate dependency coefficient, strain work-hardening exponent and thermal softening exponent, respectively. The values of these five model parameters are listed in Table 2. Moreover, The simulation of chip formation is achieved through an arbitrary Lagrangian-Eulerian (ALE) method.

$$
\bar{\sigma}=\left[A+B\left(\bar{\varepsilon}^{p l}\right)^{n}\right] \cdot\left[1+C \ln \left(\frac{\mathscr{E} p^{l}}{\&}\right)\right] \cdot\left[1-\left(\frac{T-T_{r}}{T_{m}-T_{r}}\right)^{m}\right]
$$

Table 2 The parameters of Ti-6Al-4V for J-C constitutive model

\begin{tabular}{cccccc}
\hline Material & $A(\mathrm{MPa})$ & $B(\mathrm{MPa})$ & $C$ & $n$ & $m$ \\
\hline Ti-6Al-4V & 1098 & 1092 & 0.014 & 0.93 & 1.1 \\
\hline
\end{tabular}

In section 2, three contact feature points $\left(S_{1}, S_{2}\right.$ and $\left.S_{3}\right)$ are defined through material separation theory. This study positions three feature points through $\alpha$ and Fig. 9 compares the values of $\alpha_{1}, \alpha_{2}$ and $\alpha_{3}$ between simulation and prediction. There is a good agreement between simulation and prediction although the positions of feature points change slightly with simulation time.

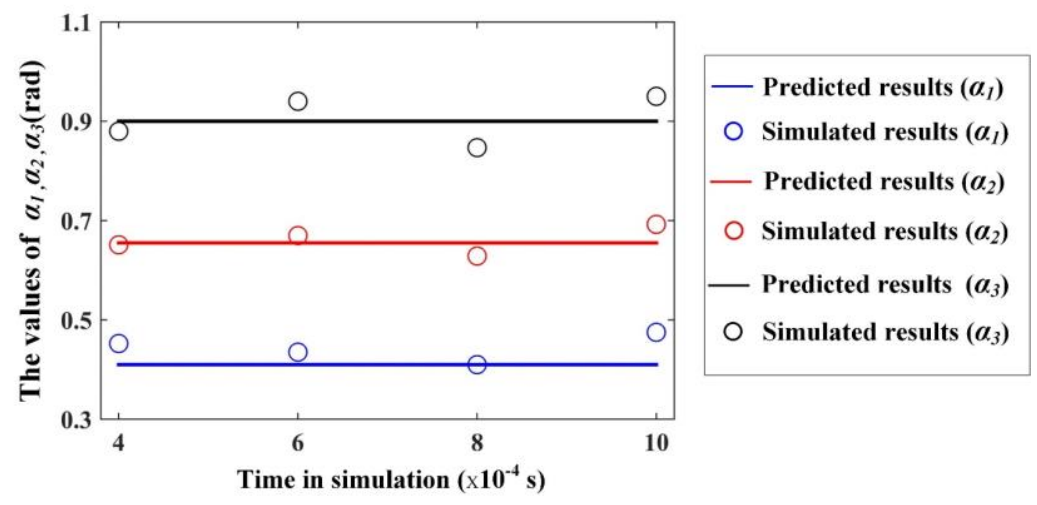

Fig. 9 The comparison of contact feature points between predicted results and simulated results

$$
(r=0.04 \mathrm{~mm}, h=0.1 \mathrm{~mm})
$$

In simulation model, the stable triangular structure attached to the round edge forms with steady cutting. $S_{1} S_{3}$ defines the radian distance between $S_{1}$ and $S_{3}$ and its value is achieved in Eq. (26). Fig. 10 gives the comparison of $S_{1} S_{3}$ between simulation and prediction and all simulation values are around the predicted values. The good agreement between numerical simulation and analytical prediction indicates the accuracy of the material separation model.

$$
S_{1} S_{3}=r \cdot\left(\alpha_{3}-\alpha_{1}\right)
$$




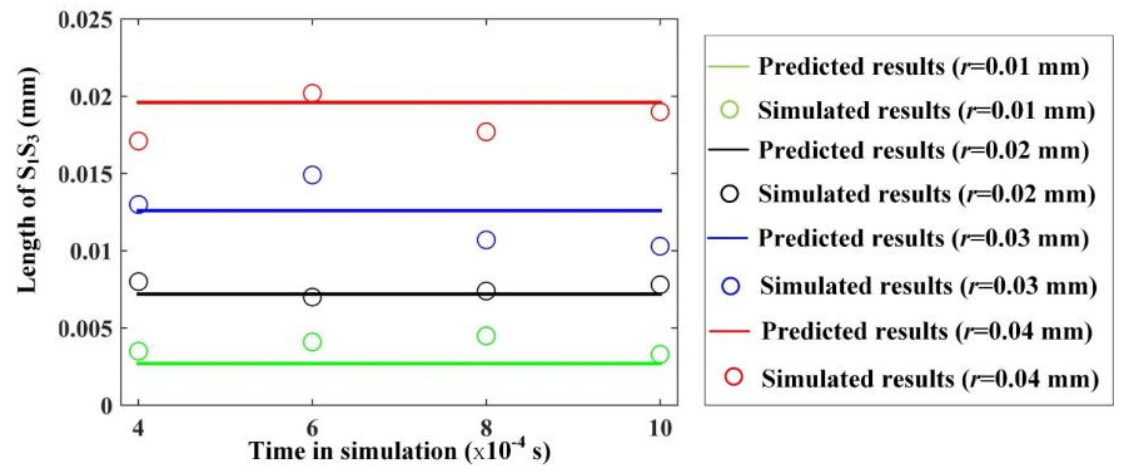

Fig. 10 The comparison of radian distance between predicted results and simulated results

The length of $\mathrm{S}_{1} \mathrm{~S}_{3}$ is the interaction length between dead metal zone and round edge. It is obvious that edge radius affects the interaction length deeply. The prediction length varies from $0.0027 \mathrm{~mm}$ to $0.196 \mathrm{~mm}$ with radius changing from $0.01 \mathrm{~mm}$ to $0.04 \mathrm{~mm}$. The conclusion is that edge radius can expand the contact region between dead metal zone and rounded edge tool.

\subsection{Validation of contact stress}

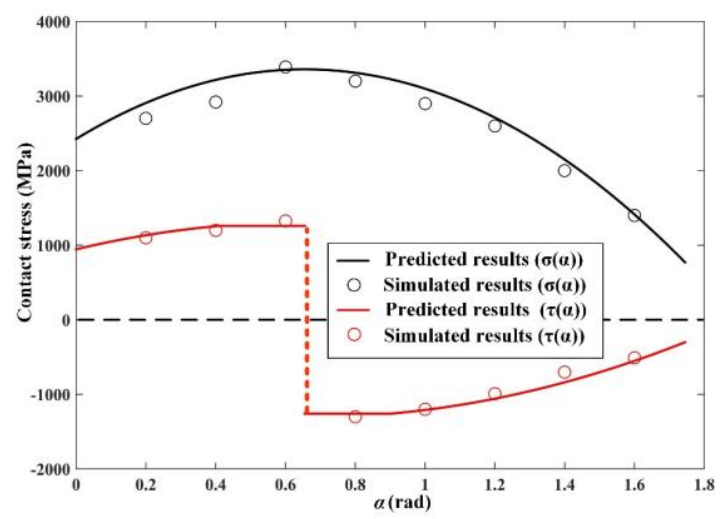

Fig. 11 The contact stress comparison between predicted results and simulated results in round

$$
\text { edge region }(r=0.04, h=0.1)
$$

In section 2, contact stress distribution between rounded edge tool and workpiece has been defined with contact feature points in detail. The contact stress comparison between proposed prediction model and finite element simulation is conducted in this section. Fig. 11 illustrates the contact stress along the round edge region. It is clearly shown that the error between prediction and simulation is relatively small, which validates the accuracy of proposed stress model. With increasing the value of $\alpha$, the normal contact stress increases firstly and then decreases nonlinearly. Besides, there exits the limiting shear stress and a sudden change of stress happens during evolution process with shear stress. 

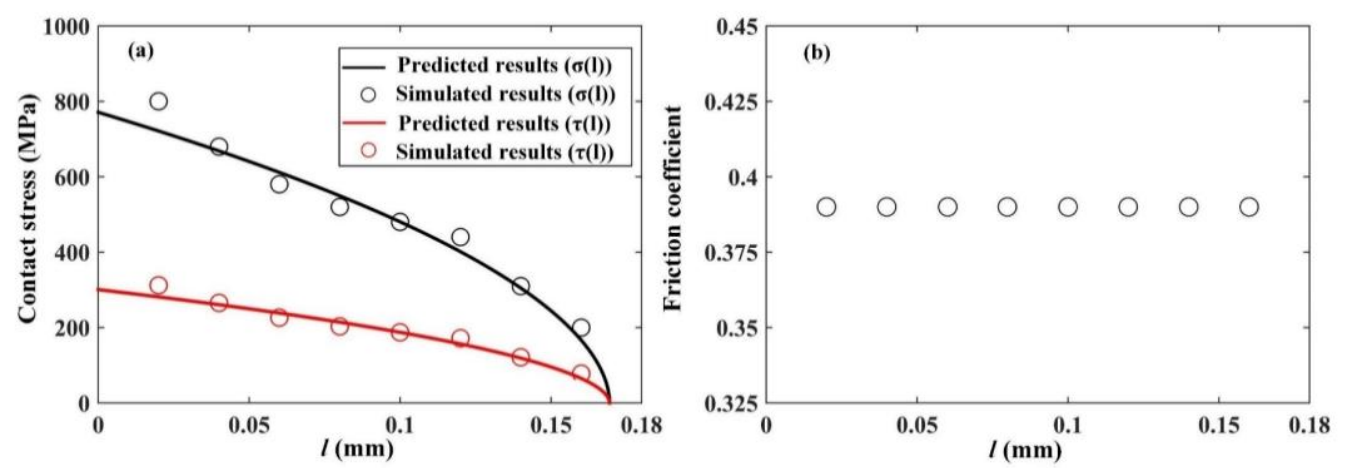

Fig. 12 Contact situation along the rake face with $r=0.04$ and $h=0.1$ (a. The co mparison between predicted results and simulated results,$b$. The frictional coefficient distribution)

Fig. 12(a) compares the contact stress along the rake face. It is obvious that all simulated stress values are around the predicted value both in normal and shear direction. The small error between simulation and prediction ensures the accuracy of proposed contact model in rake face. Besides, the friction coefficient along the rake face is also explored shown in Fig. 12(b). The value of coefficient keeps constant at 0.39 , which proves the thought that the rake face region belongs to the sliding region.

\subsection{Validation with published results}

Wyen and Wegener [27] have presented measured forces from orthogonal turning tests conducted on Ti-6Al-4V. In their research, the Gaussian fitted circle was used to character rounded edge tools and the radius uncertainty is $2 \%$ of the diameter. The cutting force data with different edge radius and feeds were measured through a Kistler 9121 dynamometer. As for the experiment setup, the cutting tools were made of $\mathrm{WC} / \mathrm{Co}$. The rake angle was $10^{\circ}$ and clearance angle was selected as $8^{\circ}$. The edge radius varied from $0.01 \mathrm{~mm}$ to $0.05 \mathrm{~mm}$ and the specific cutting edge geometry was recognized by an Alicona Infinite Focus device. Besides, uncut chip thickness varied from $0.01 \mathrm{~mm}$ to $0.2 \mathrm{~mm}$ and cutting width was $2 \mathrm{~mm}$.

Fig. 13 compares cutting forces between experiment by Wyen and Wegener and prediction by proposed analytical method with various parameters. There is a good agreement between prediction and experiment, which proves the accuracy and feasibility of proposed analytical model. Besides, the force error in feed direction is relatively smaller than it in cutting direction. When $r=0.04 \mathrm{~mm}$ and $h=0.1 \mathrm{~mm}$, there is a force deviation both in cutting and feed direction. This deviation expresses the shortage of the proposed model when the ratio of the edge radius to the uncut chip thickness is large. The effects edge radius on cutting forces is also displayed in Fig. 13. It is clear that both $F_{t}$ and $F_{f}$ increase with increasing edge radius. However, edge radius affects $F_{f}$ more largely than $F_{t}$. 

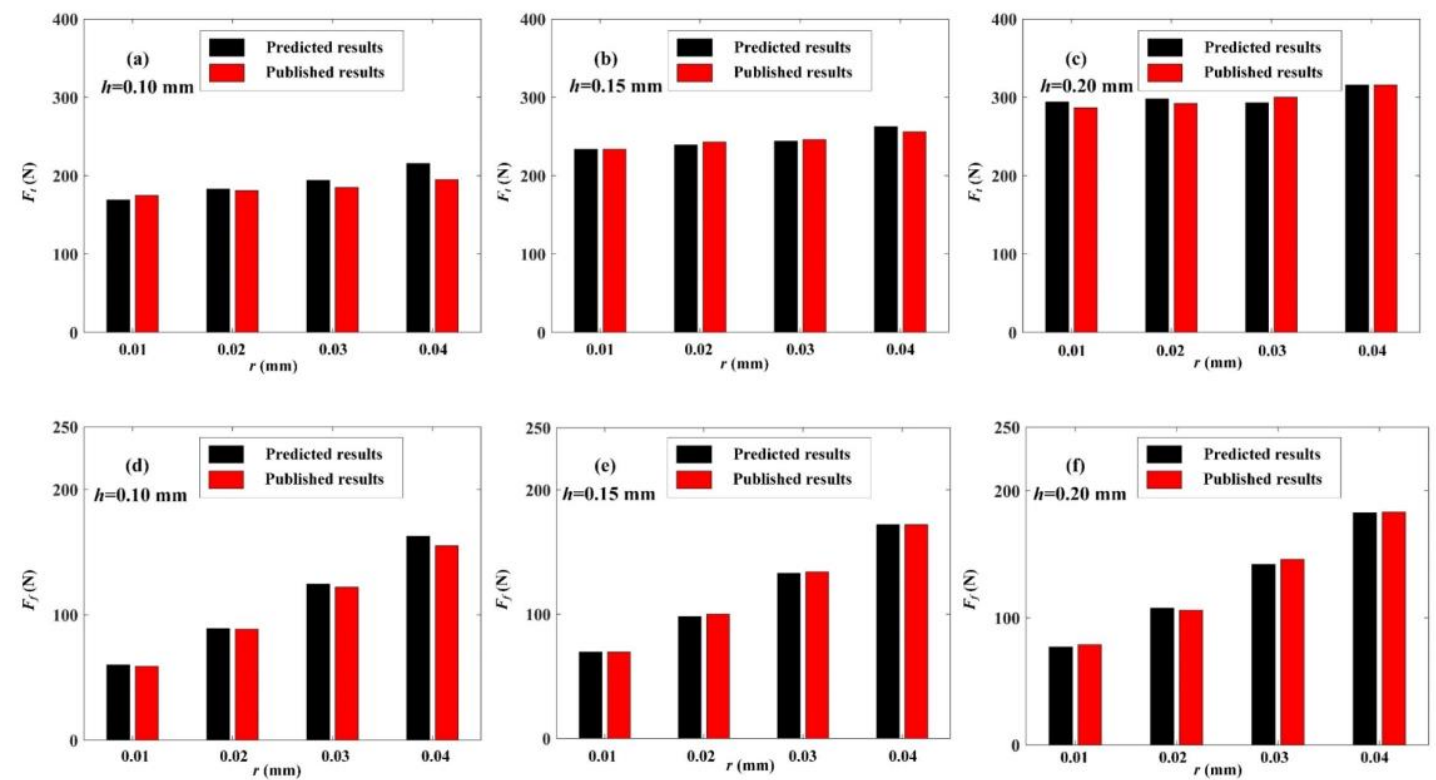

Fig. 13 The force comparison between predicted results and published results

\subsection{Validation with experimental results}

Series of orthogonal cutting tests have been illustrated to validate the accuracy of the predicted method. Fig. 14 shows the experimental setup, the cutting material is Ti-6Al-4V bar, which is processed in advance to produce grooves for orthogonal cutting. The tool used in cutting tests is carbide insert and the tool holder is also displayed in Fig. 14. The rake angle and clearance angle are $10^{\circ}$ and $8^{\circ}$, respectively. Besides, the type number of the lathe is selected as CAK5085nzj and cutting forces are measured through Kistler 9257B dynamometer. The cutting data acquisition frequency is $40 \mathrm{kHz}$. As for the selection of the cutting parameters, the cutting speed is $70 \mathrm{~m} / \mathrm{min}$, feed varies from $0.06 \mathrm{~mm} / \mathrm{r}$ to $0.15 \mathrm{~mm} / \mathrm{r}$.

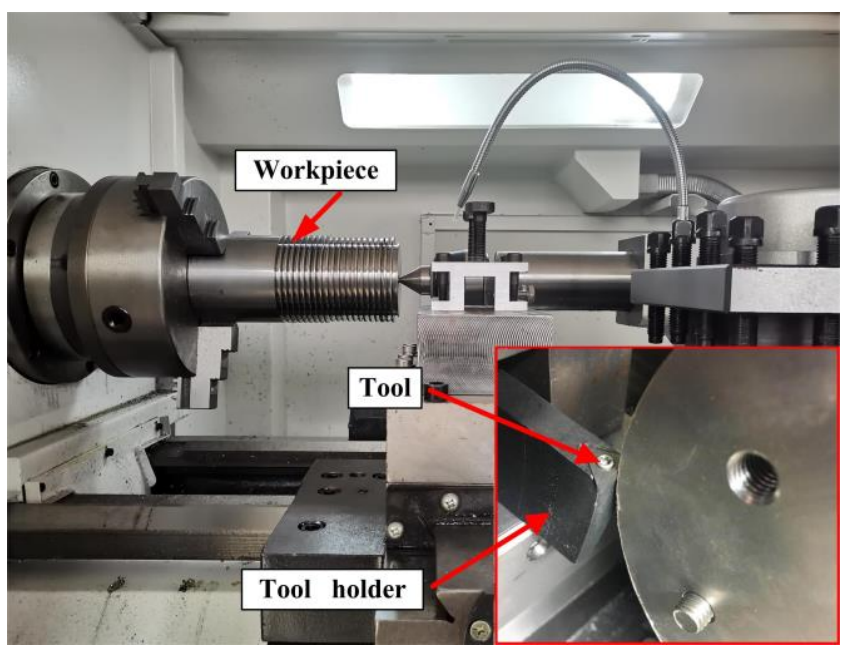

Fig. 14 The experiment setup 

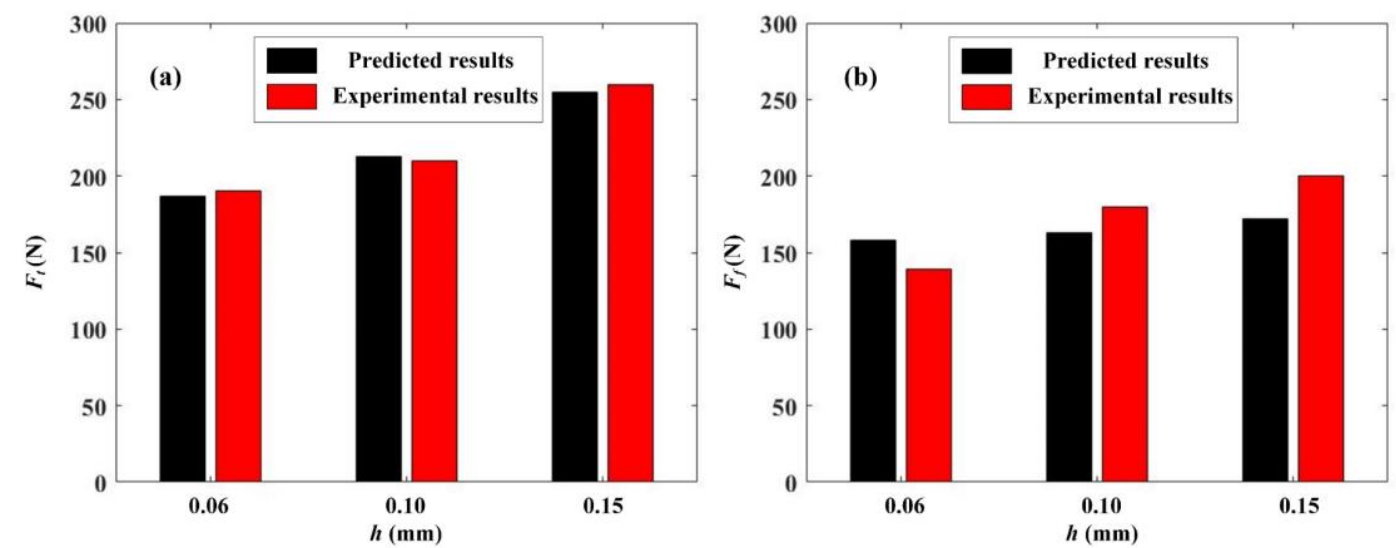

Fig. 15 The forces comparison between predicted results and experimental results with $v=70$ $\mathrm{m} / \mathrm{min}$ and $r=0.04 \mathrm{~mm}$ (a. Forces in cutting direction, $\mathrm{b}$. Forces in feed direction)

Fig. 15 compares the cutting forces between experimental and analytical prediction. It can be seen that both $F_{t}$ and $F_{f}$ increase with enlarging the uncut chip thickness. Besides, the increasing trend of $F_{t}$ is larger than $F_{f}$. It is clear that there exists a good agreement between the experimental and predicted results. Therefore, the proposed contact model is suitable in predicting cutting forces considering round edge.

The insert surface morphology after orthogonal cutting is identified by microscope as shown in Fig. 16 (a). It should be noted that there are lots of sliding marks resulted from sliding chip in the rake face. Fig. 16 (b) compares the values of contact length between the experiment measurement and model prediction. The error between experiment and prediction is relatively small, which proves the accuracy of the proposed contact model.
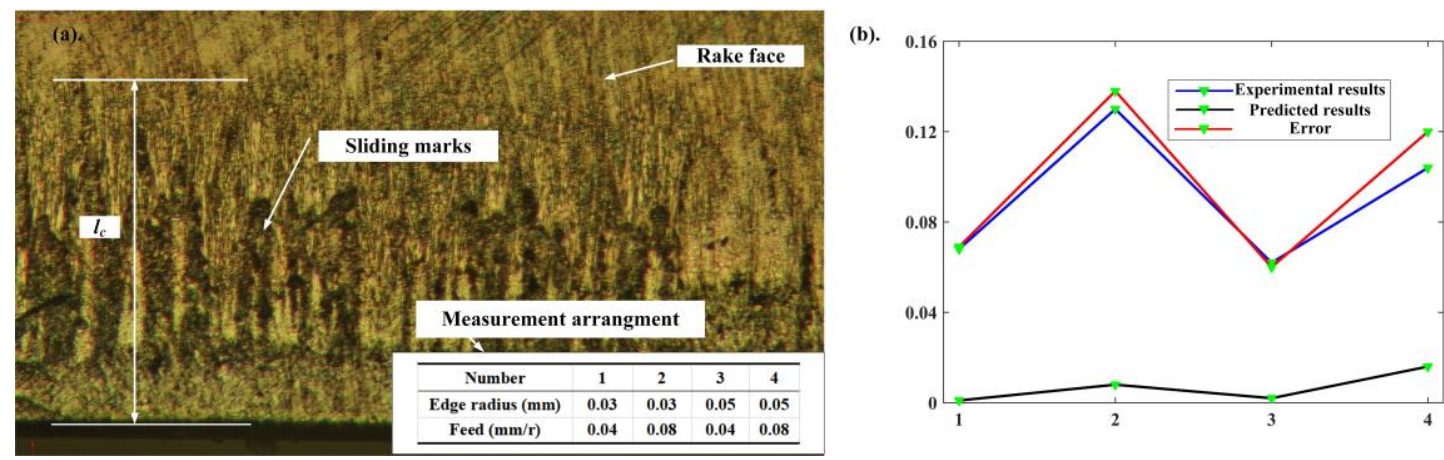

Fig. 16 Contact length prediction in orthogonal cutting with carbide tool

(a. The insert surface morphology in rake face; b. The contact length of experiment and prediction)

Alicona EdgeMasterModule 6.5 is used to identify the round edge shape as shown in Fig. 17. The edge shapes of insert both before cutting and after cutting are obtained. It is found that the wear of edge occurs after cutting. Besides, the wear mainly occurs around the point $S_{2}$, which proves the assumption that the maximum stress happens at the point $S_{2}$. 


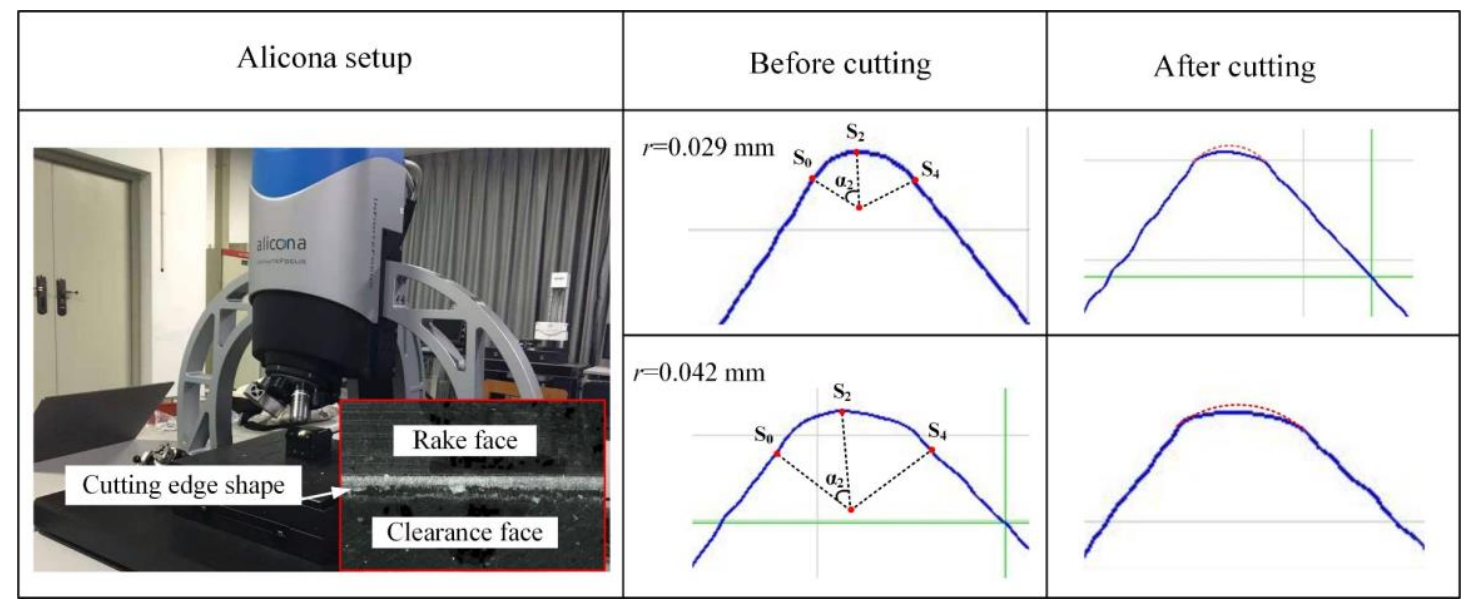

Fig. 17 The edge wear in orthogonal cutting

\section{Results and discussion}

This study presents a developed contact stress model considering dead metal zone in cutting with rounded edge tool. Section 2 establishes the contact model in detail and some model variables are determined with experimental force data in section 3 . Then this section explores contact stress and cutting forces affected by edge radius in different contact regions.

\subsection{Contact stress distribution}

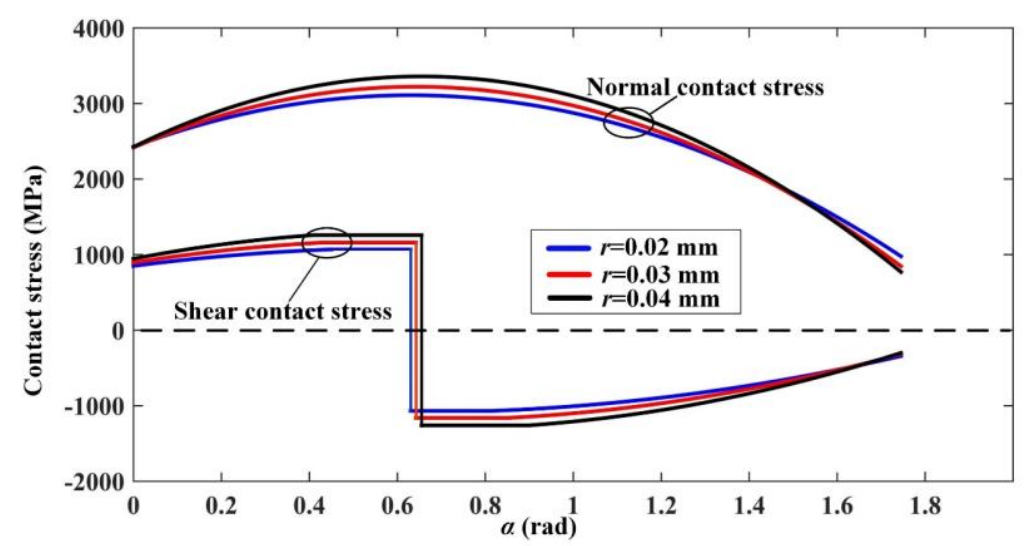

Fig. 18 Contact stress distribution along the round edge with the proposed model $\left(\mathrm{S}_{0}-\mathrm{S}_{4}\right)$

Fig. 18 offers the contact stress along round edge. As defined in section 2.1, the distribution function of normal contact stress is a parabola resulted from $\alpha$. There exists maximum normal contact stress along the round edge and the maximum stress increases with enlarging the edge radius. As for shear contact condition, the shear limiting stress occurs in the round edge region. The value of the limiting stress increases with enlarging the edge radius. In fact, edge radius contributes to the overall shear stress distribution in the round edge. Besides, there is a sudden change in the direction during shear contact stress. This cliff change confirms the existence of point $S_{2}$. It is necessary to point out that the ratio of edge radius to uncut chip thickness affects the round edge contact situation. However, the parameter selection in this research ensures that the round edge area is in full contact with the workpiece. In this situation, the contact behavior along the round edge is independent with uncut chip thickness. 


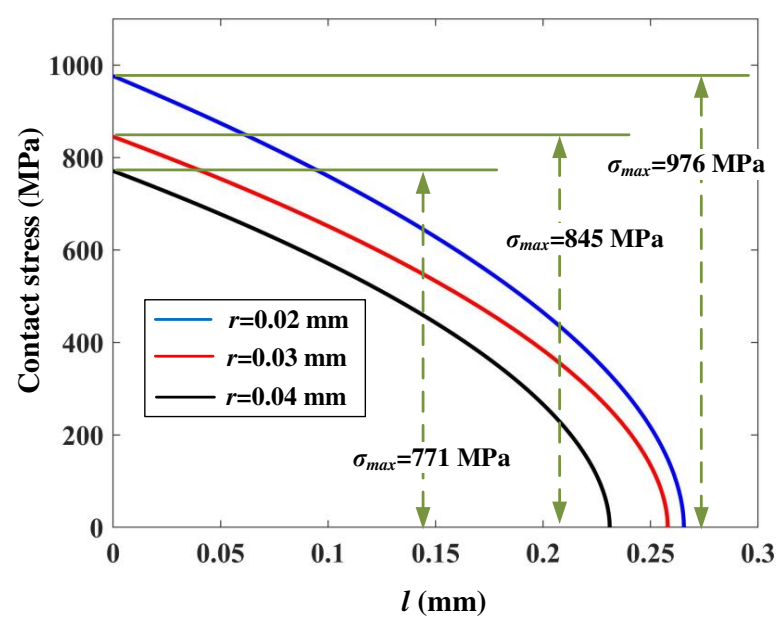

Fig. 19 Normal contact stress along the rake face $\left(\mathrm{S}_{4}-\mathrm{S}_{5}\right)$

$$
(h=0.10 \mathrm{~mm})
$$

The contact stress distribution along the rake face is shown in Fig. 19. As expressed in Eq. (13), the maximum normal contact stress $\left(\sigma_{\max }\right)$ and contact length $\left(l_{c}\right)$ are two important variables determining the stress distribution. The increase of edge radius can restrain maximum normal stress in the rake face region.

In cutting with rounded edge tool, edge radius largely affects both the normal and shear contact behavior. Besides, not only the contact situation along the round edge is influenced by edge radius but also contact behavior of rake face is dependent on edge radius.

\subsection{Tool-based force components}
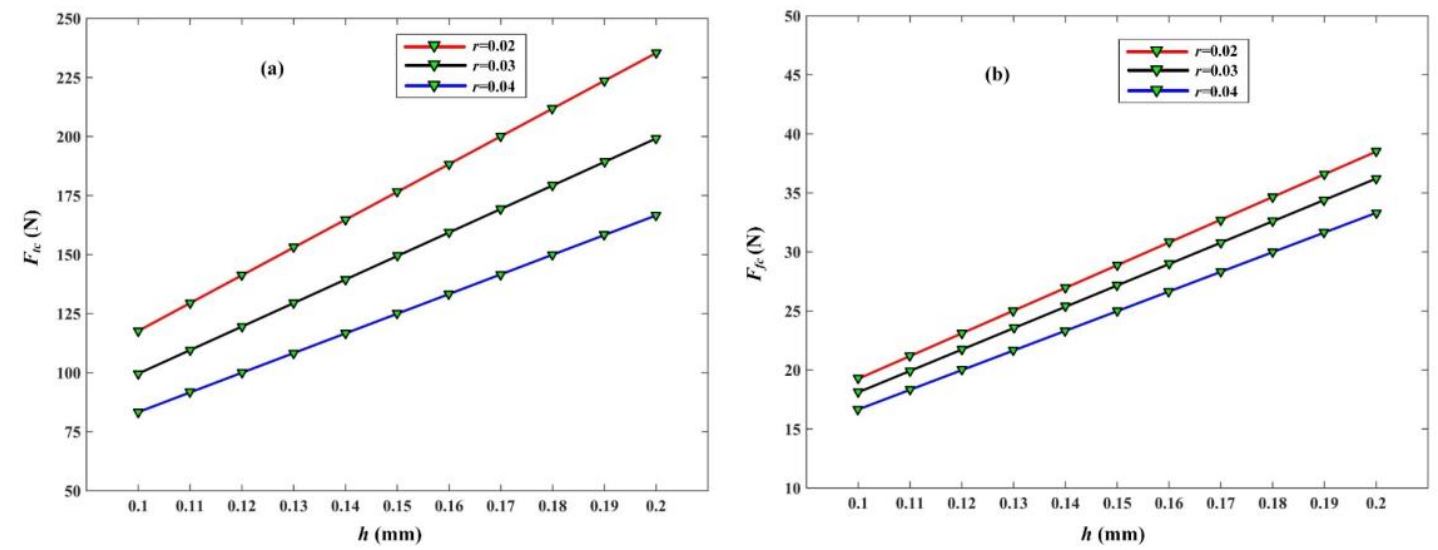

Fig. 20 Contact force component along the rake face

(a. $F_{c}$ in cutting direction, b. $F_{c}$ in feed direction)

This study explores cutting forces based on contact force analysis in section 2 and the whole cutting forces include two contact force components expressed in Eq. (15). Fig. 20 displays contact forces along the rake face. It is clear that both $F_{t c}$ and $F_{f c}$ increase with enlarging the uncut chip thickness. Besides, increasing edge radius can largely reduce $F_{t c}$ while the influence of edge radius on $F_{f c}$ is relatively small. 

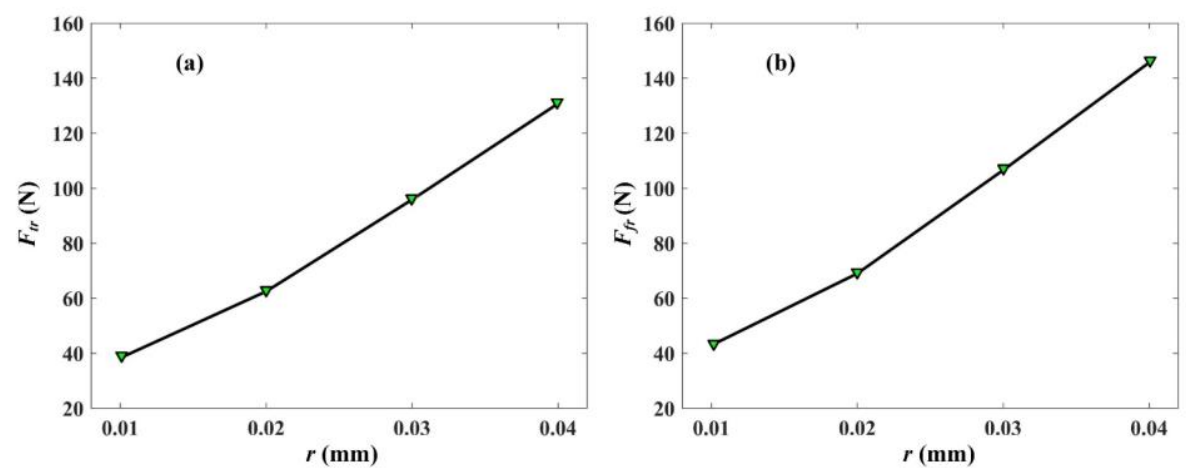

Fig. 21 Contact force component along the round edge

(a. $F_{r}$ in cutting direction, b. $F_{r}$ in feed direction)

The contact force components in the round edge are researched in Fig. 2. Both $F_{t r}$ and $F_{f r}$ increase with edge radius increasing. It should be noted that the contact situation in round edge is assumed to be constant with uncut chip thickness changing once chip interacts with rake face in this study. Thus, the values of $F_{t r}$ and $F_{f r}$ achieved with $h=0.1 \mathrm{~mm}$ to $0.2 \mathrm{~mm}$ are the same in this research.

It is known that both $F_{t}$ and $F_{f}$ increase with enlarging the edge radius. The change magnitude of $F_{f}$ is much more than that of $F_{t}$, which means the value of $F_{f}$ is more sensitive to changing edge radius. When edge radius is enlarged, the decrease of $F_{t c}$ and the increase of $F_{t r}$ can cancel each other. As a result, the effect of edge radius on $F_{t}$ is relatively little. As for $F_{f}$, with increasing edge radius, it is the substantial increase of $F_{f r}$ which increases $F_{f}$.

\section{Conclusions}

This study introduces tool-based cutting forces with the contact behavior exploration along the round edge. A contact stress distribution model with exploring contact feature points is established. The important points are shown as follows,

Firstly, three contact feature points are defined in the round edge with the consideration of plough mechanism. $S_{1}$ and $S_{3}$ are two points where dead metal zone boundary contacts with round edge and $S_{2}$ is the point where shear contact stress is zero. The boundary of dead metal zone expands with increasing edge radius.

Secondly, the normal contact stress along the round edge is set as a parabolic equation and increasing edge radius can enlarge the normal stress. Besides, the shear limiting stress occurs along the round edge and its value increases with enlarging the edge radius.

Thirdly, the tool-based contact force model is established with contact stress distribution. The total cutting force is divided into force components of rake face and round edge. Increasing edge radius can increase the total cutting forces both in cutting and feed direction. As for the force components in rake face, when edge radius increases, the force component in cutting direction decreases, while the component in feed direction changes slightly. What's more, in round edge contact region, the force components both in cutting and feed direction increase with enlarging edge radius.

In this study, the contact stress model is based on the premise that the tool-workpiece contact between rake face and workpiece occurs. However, when the ratio of uncut chip thickness to edge radius is relatively small, the contact only happens between round edge and workpiece. The systematical contact exploration with little ratio will be achieved in further research. 
Author contribution Weiwei Zhang: Writing original draft, methodology, validation and investigation. Jian Weng: Writing-review \& editing, validation. Kejia Zhuang: Supervision, writing-review and editing, review of experimental setup. Cheng Hu: Investigation. Xing Dai: Investigation. Chaoqun Wu: Supervision, investigation.

Funding This work is partially supported by the National Natural Science Foundation of China (51705385, 51975237), and State Key Laboratory of Digital Manufacturing Equipment and Technology (DMETKF2020009).

Availability of data and material The authors confirm that the data supporting this study are available within the article.

Code availability Not applicable.

\section{Declarations}

Ethics approval The manuscript has not been submitted to any other journal for simultaneous consideration. The submitted work is original and has not been published elsewhere in any form or language.

Consent to participate All authors voluntarily agree to participate in this research study.

Consent for publication All authors voluntarily agree to the publication of the paper.

Conflict of interest The authors declare no conflict of interest.

\section{References}

[1] Ulutan D, and Özel T (2013) Determination of tool friction in presence of flank wear and stress distribution based validation using finite element simulations in machining of titanium and nickel based alloys. J Mater Process Tech 213 (12): 2217-2237.

[2] Zhuang K, Weng J, Zhu D, and Ding H (2018) Analytical modeling and experimental validation of cutting forces considering edge effects and size effects with round chamfered ceramic tools. ASME J Manuf Sci E 140 (8): 081012.

[3] Yang S, Ren W, Wang T, and Su S (2020) Parameter optimization of a micro-textured ball-end milling cutter with blunt round edge. Int J Adv Manuf Tech 106 (1): 577-588.

[4] De Oliveira FB, Rodrigues AR, Coelho RT, and De Souza AF (2015) Size effect and minimum chip thickness in micromilling. Int J Mach Tool Manu 89: 39-54.

[5] Przestacki D, Chwalczuk T, and Wojciechowski S (2017) The study on minimum uncut chip thickness and cutting forces during laser-assisted turning of WC/NiCr clad layers. Int J Adv Manuf Tech 91 (9-12): 3887-3898.

[6] Albrecht P (1960) New developments in the theory of the metal-cutting process: Part I. The ploughing process in metal cutting. J Eng Ind 82 (4): 348-357. 
[7] Waldorf DJ, DeVor RE, and Kapoor SG (1998) A slip-line field for ploughing during orthogonal cutting. ASME J Manuf Sci E 120 (4): 693-699.

[8] Fang N (2003) Slip-line modeling of machining with a rounded-edge tool-Part II: analysis of the size effect and the shear strain-rate. J Mech Phys Solids 51 (4): 743-762.

[9] Uysal A, and Altan E (2016) Slip-line field modelling of rounded-edge cutting tool for orthogonal machining. P I Mech Eng B-J Eng 230 (10): 1925-1941.

[10] Hu C, Zhuang K, Weng J, Zhang X, and Ding H (2020) Cutting temperature prediction in negative-rake-angle machining with chamfered insert based on a modified slip-line field model. Int $\mathrm{J}$ Mech Sci 167: 105273.

[11] Hu C, Zhuang K, Weng J, and Zhang X (2019) Thermal-mechanical model for cutting with negative rake angle based on a modified slip-line field approach. Int J Mech Sci 164: 105167.

[12] Jin X, and Altintas Y (2012) Prediction of micro-milling forces with finite element method. J Mater Process Tech 212 (3): 542-552.

[13] Zhou T, He L, Zou Z, Du F, Wu J, and Tian P (2020) Three-dimensional turning force prediction based on hybrid finite element and predictive machining theory considering edge radius and nose radius. J Manuf Process 58: 1304-1317.

[14] Arrazola PJ, Ugarte D, and Domínguez X (2008) A new approach for the friction identification during machining through the use of finite element modeling. Int J Mach Tool Manu 48 (2): 173-183.

[15] Zhang W, Zhuang K, and Pu D (2020) A novel finite element investigation of cutting force in orthogonal cutting considering plough mechanism with rounded edge tool. Int J Adv Manuf Technol 108 (9-10): 3323-3334.

[16] Laakso SVA, Agmell M, and Ståhl J-E (2018) The mystery of missing feed force — The effect of friction models, flank wear and ploughing on feed force in metal cutting simulations. J Manuf Process 33: $268-277$.

[17] Woon KS, Rahman M, Neo KS, and Liu K (2008) The effect of tool edge radius on the contact phenomenon of tool-based micromachining. Int J Mach Tool Manu 48 (12): 1395-1407.

[18] Wang Y, Zou B, Huang C, Qi H, and Song J (2019) Feasibility study of the Ti (C7N3)-based cermet micro-mill based on dynamic fatigue behavior and modeling of the contact stress distribution on the round cutting edge. Int J Mech Sci 155: 143-158.

[19] Basuray PK, Misra BK, and Lal GK (1977) Transition from ploughing to cutting during machining with blunt tools. WEAR 43 (3): 341-349.

[20] Yuan ZJ, Zhou M, and Dong S (1996) Effect of diamond tool sharpness on minimum cutting thickness and cutting surface integrity in ultraprecision machining. J Mater Process Tech 62 (4): 327 330 .

[21] Liu Z, Shi Z, and Yi W (2013) Definition and determination of the minimum uncut chip thickness of microcutting. Int J Adv Manuf Technol 69 (5-8): 1219-1232.

[22] Câmara MA, Abrão AM, Rubio JCC, Godoy GCD, and Cordeiro BS (2016) Determination of the critical undeformed chip thickness in micromilling by means of the acoustic emission signal. Precis Eng 46: 377-382.

[23] Wan M, Wen D, Ma Y, and Zhang W (2019) On material separation and cutting force prediction in micro milling through involving the effect of dead metal zone. Int J Mach Tool Manu 146: 103452 .

[24] Li X, Shi Z, Duan N, Cui P, Zhang S, and Zhang X (2020) Cutting performance investigation based on the variable friction model by considering sliding velocity and limiting stress. P I Mech Eng B- 
J Eng 234 (8): 1113-1123.

[25] Dewhurst P, and Collins I (2010) A matrix technique for constructing slip-line field solutions to a class of plane strain plasticity problems. Int J Numer Meth Eng 7 (3): 357-378.

[26] Zhang W, Zhang L, Wang B, and Wang S (2019) Finite element simulation analysis of bionic ball-end milling cutter. Int J Adv Manuf Tech 103 (5): 3151-3161.

[27] Wyen C-F, and Wegener K (2010) Influence of cutting edge radius on cutting forces in machining titanium. Cirp Ann -Manuf Techn 59 (1): 93-96. 
Figures
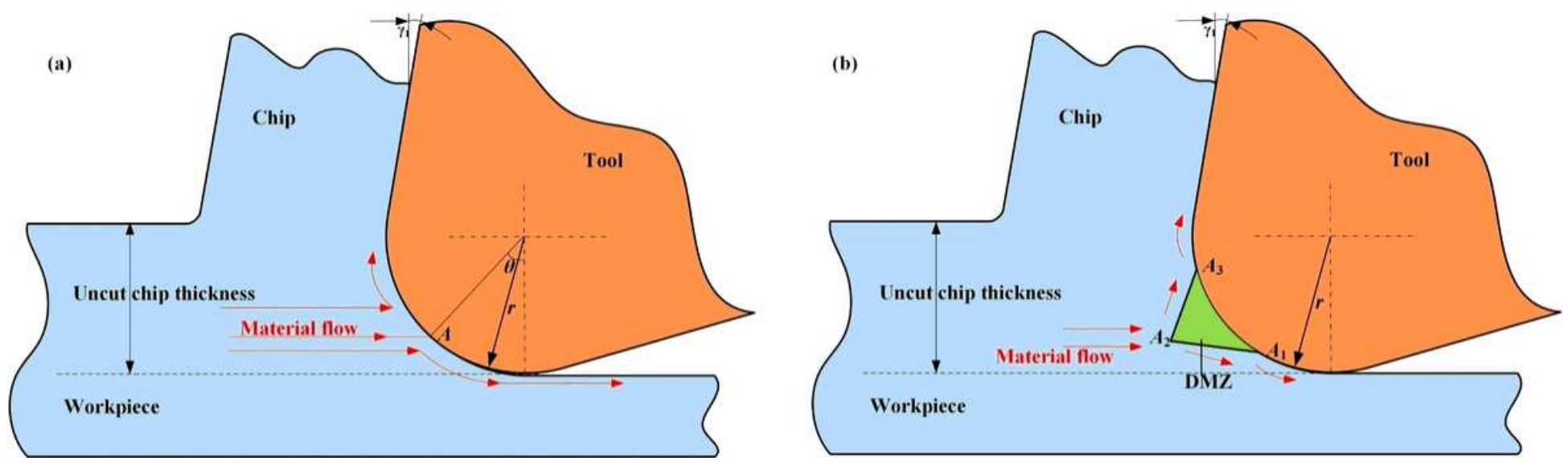

Figure 1

The classical material separation theory (a. Material separation proposed by Basuray et al. [20], b. Material separation based on DMZ [7])

\section{Chip}

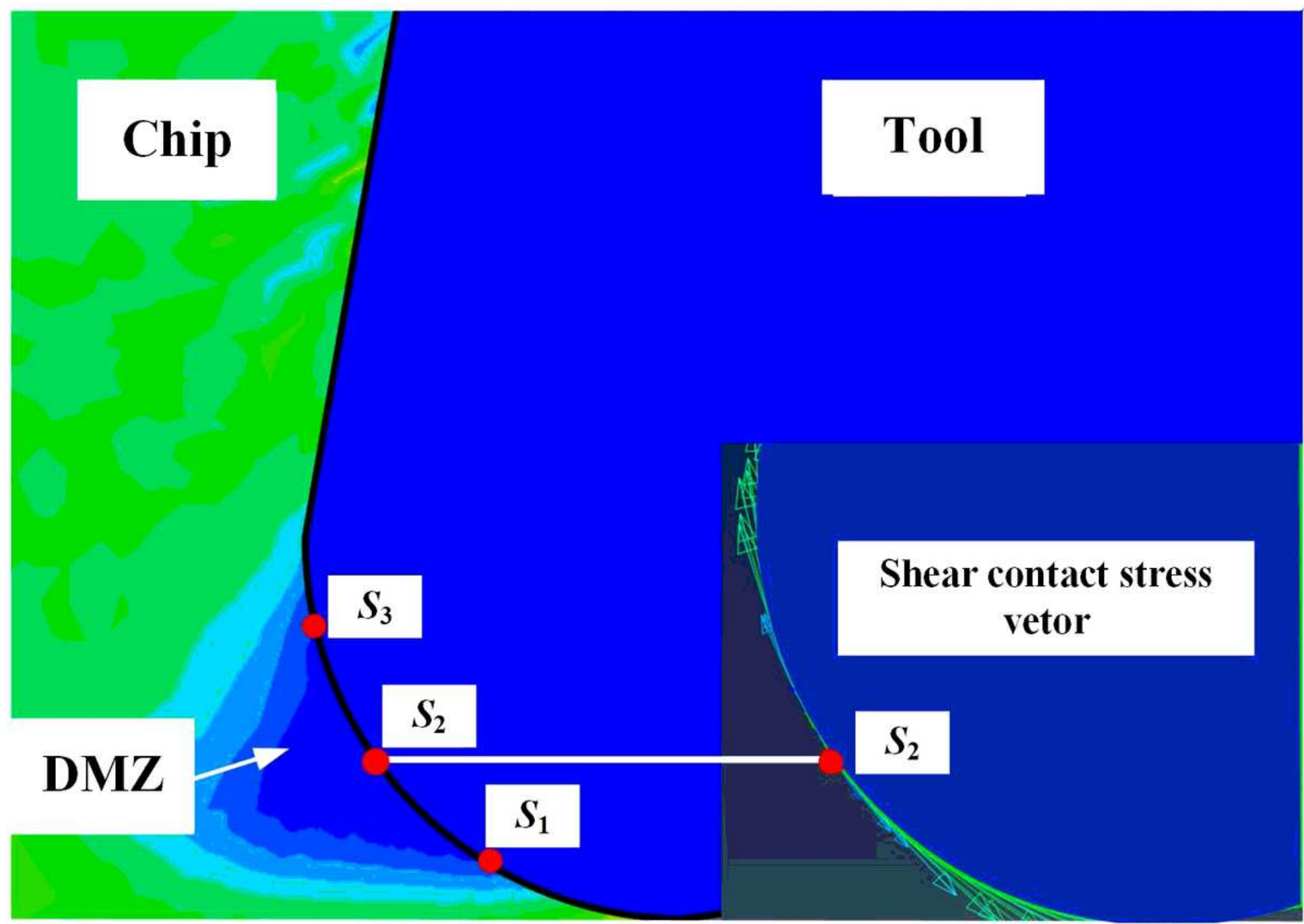


Figure 2

Tool-workpiece contact modeling considering round edge

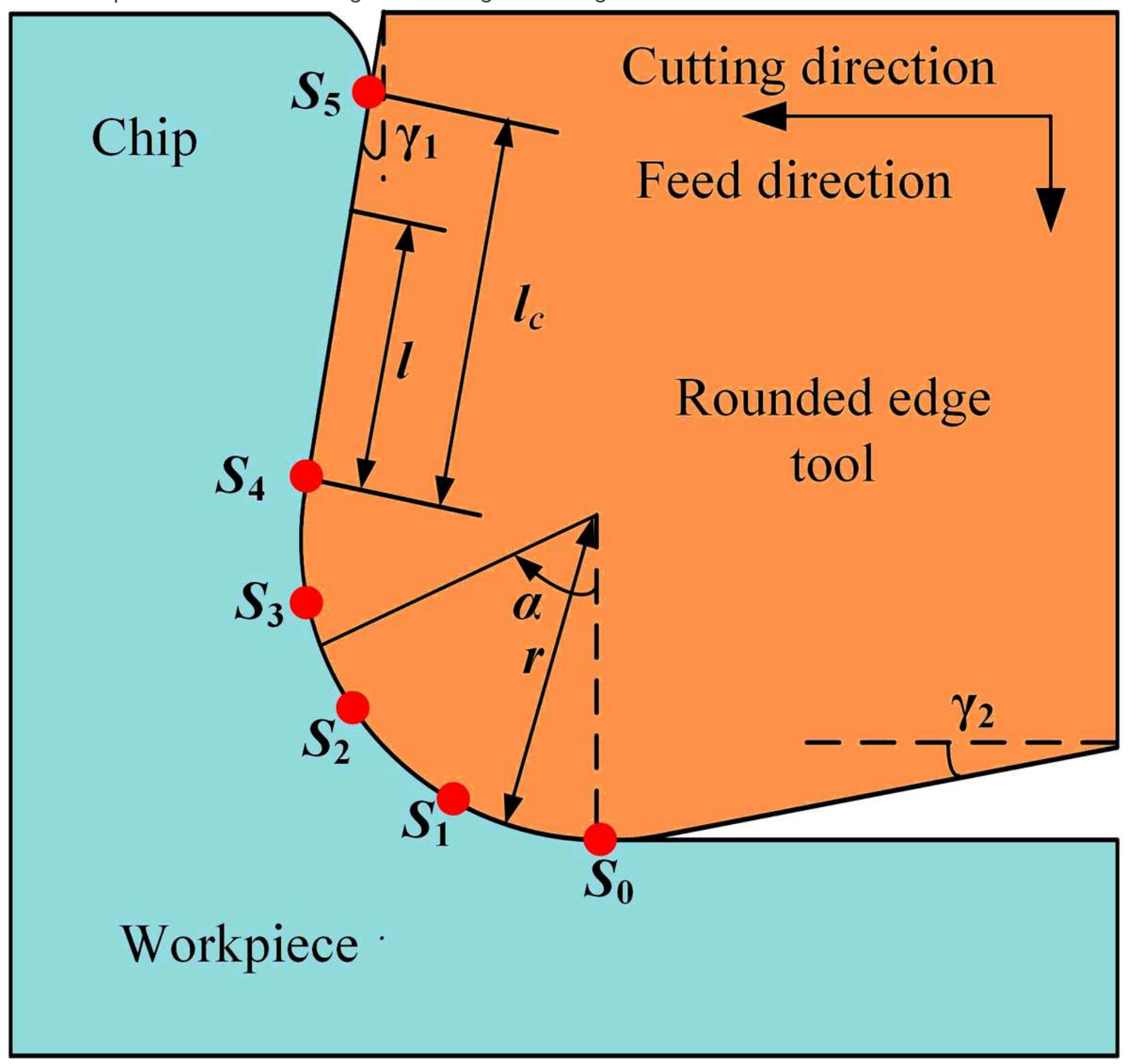

Figure 3

Contact stress points defined with round edge 


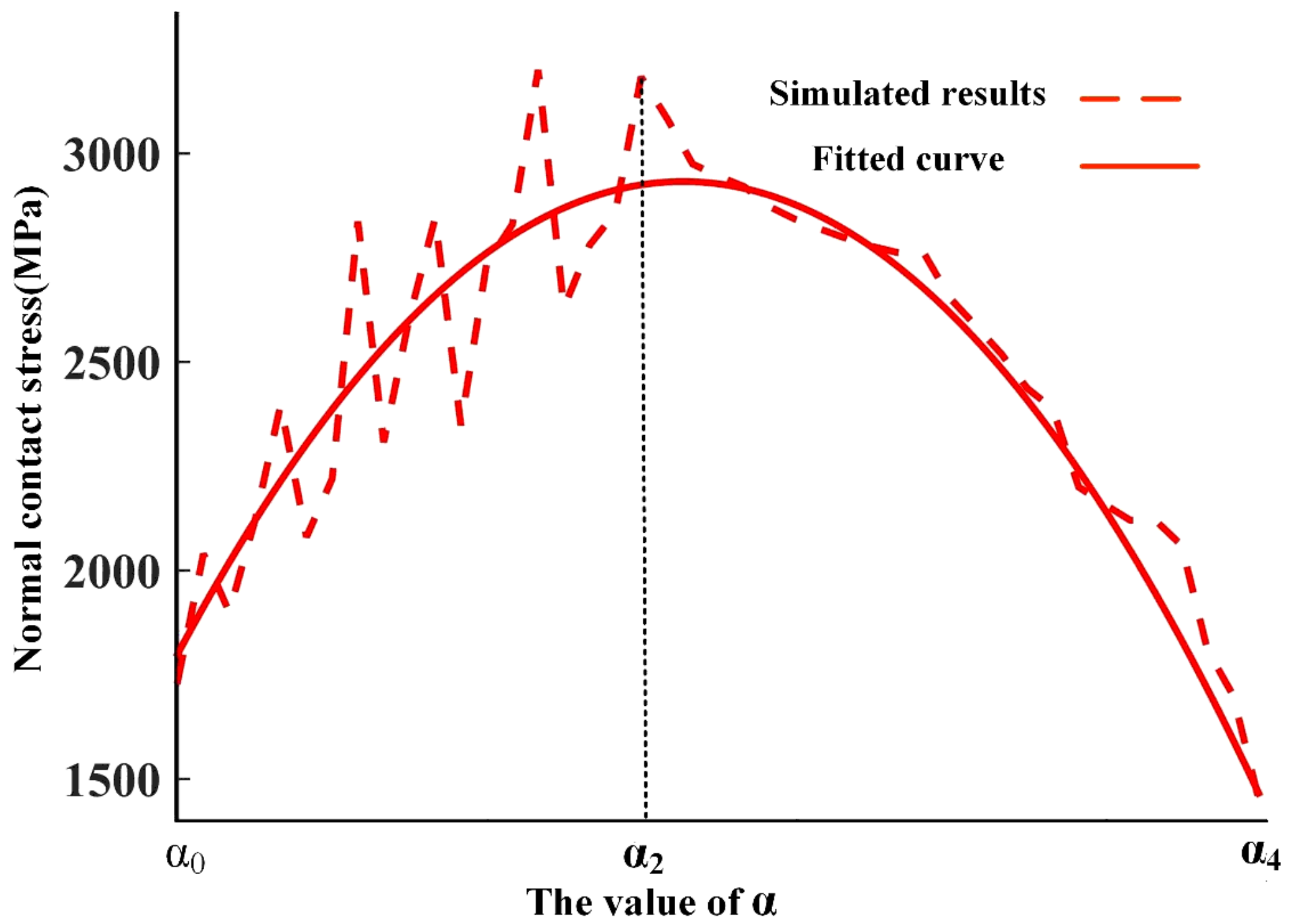

Figure 4

Normal contact stress modeling through simulation and regression

(a)

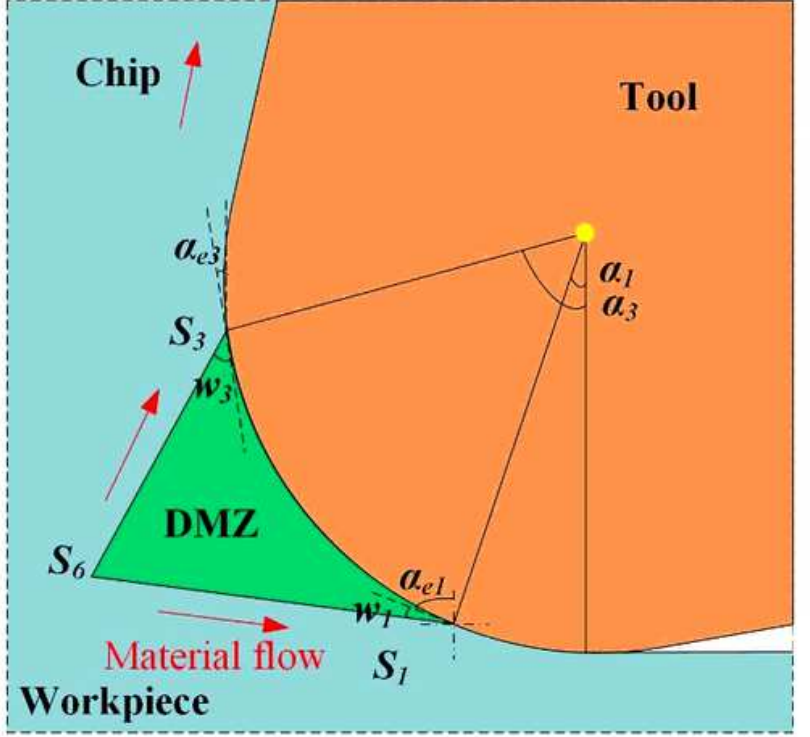

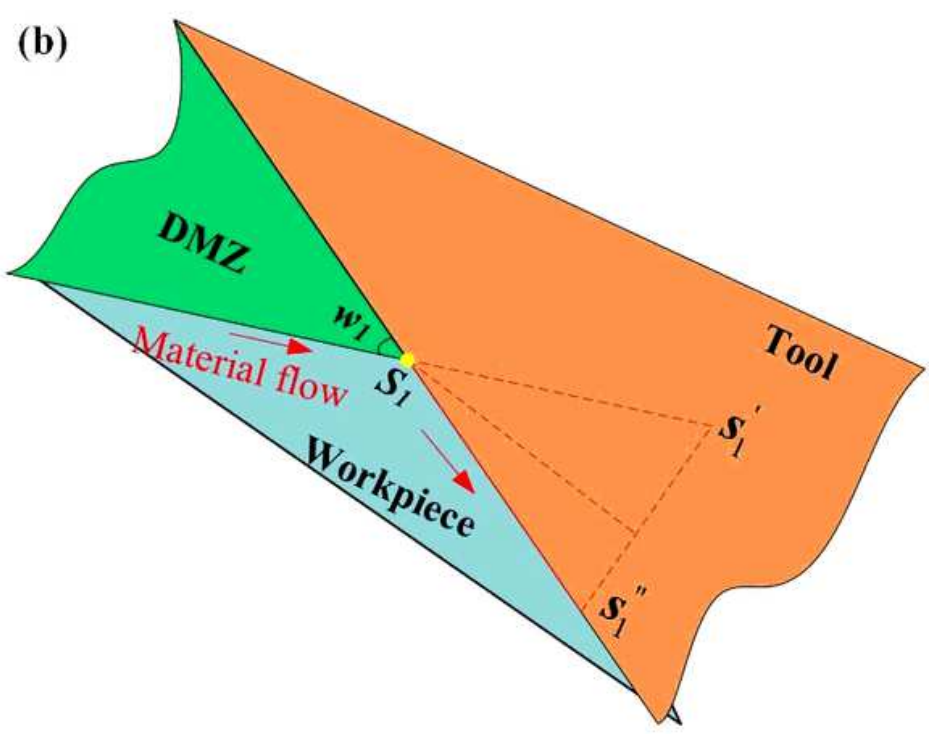

Figure 5 


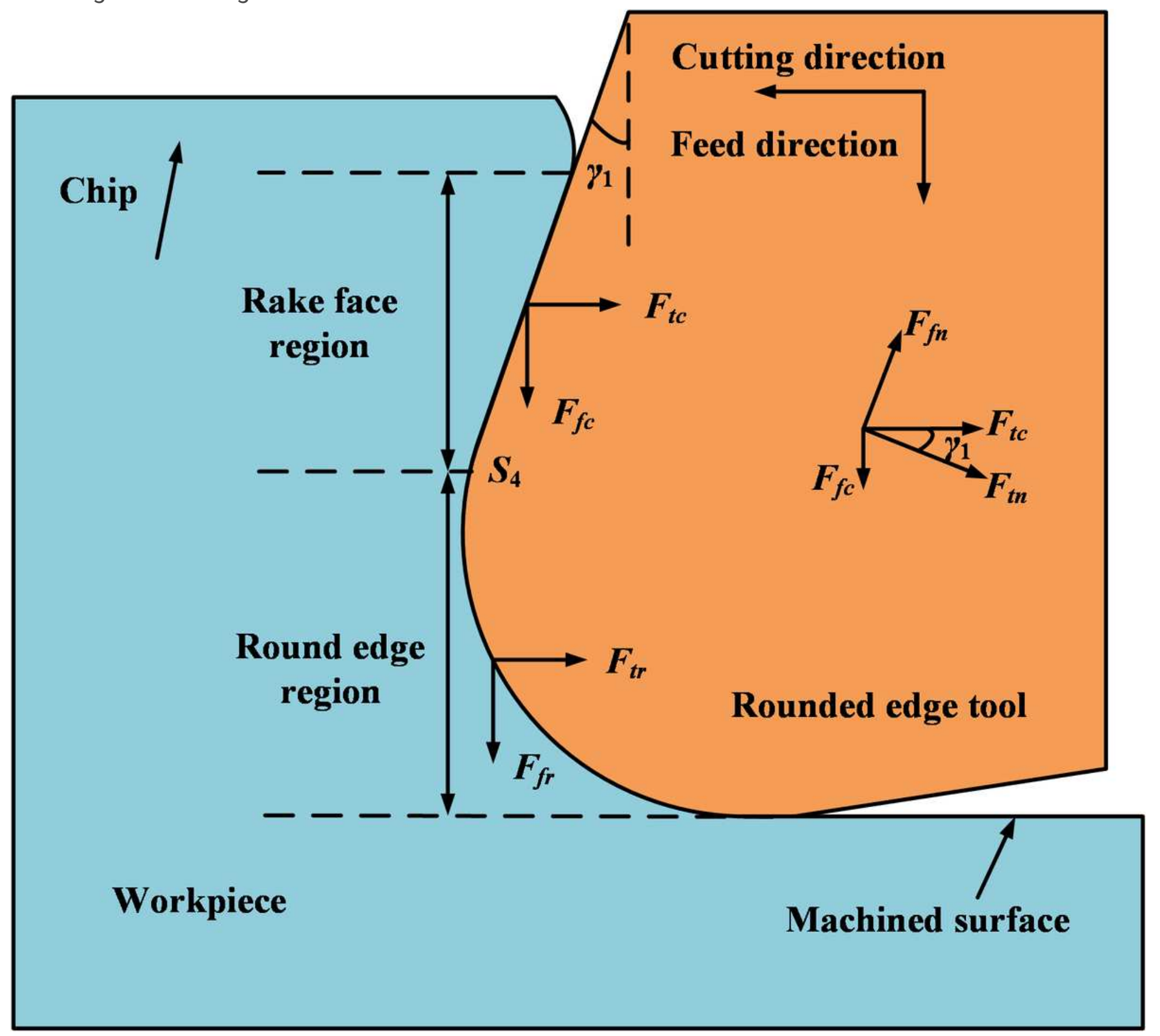

Figure 6

Force components in cutting with rounded edge tool 


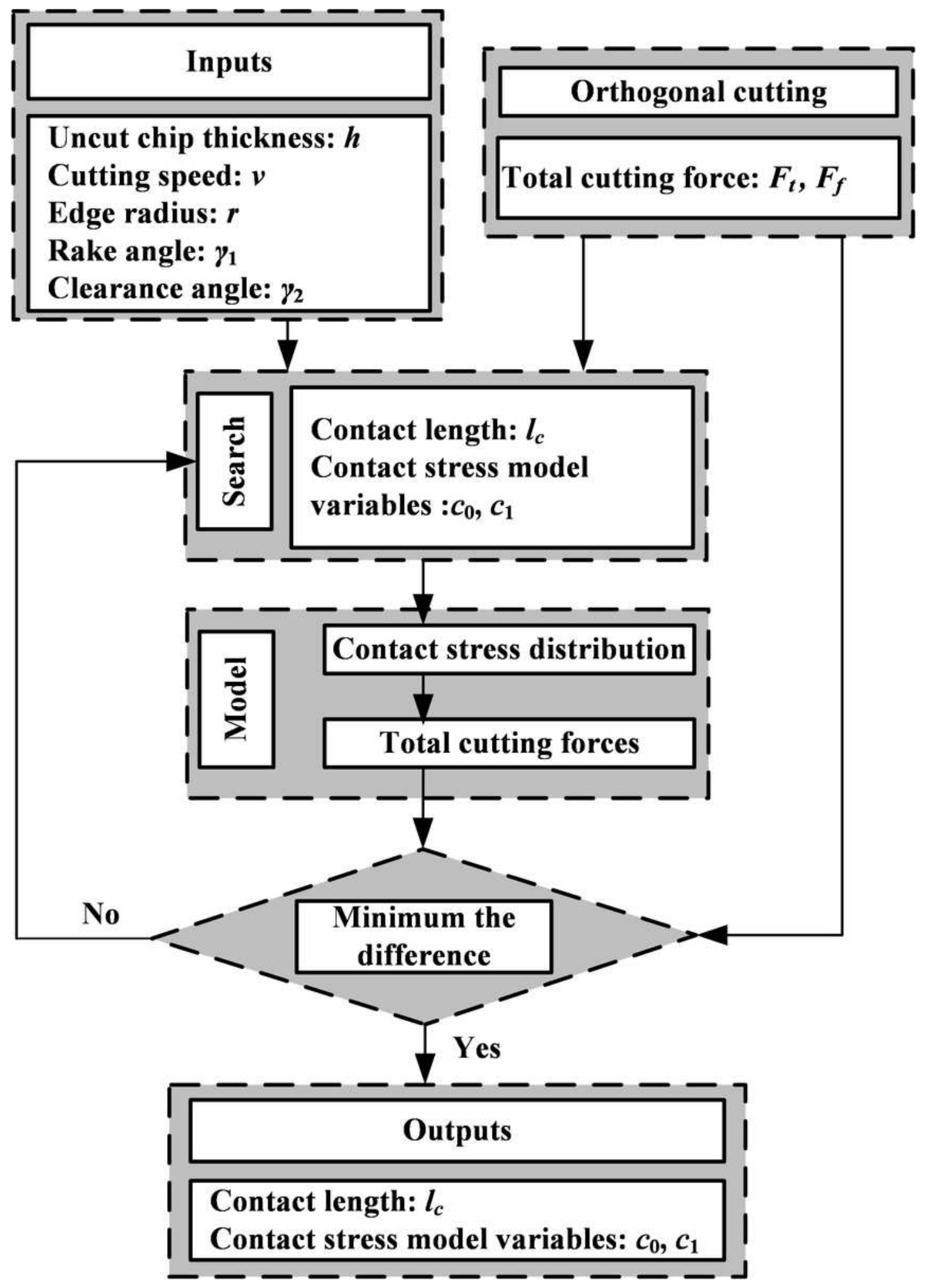

Figure 7

Illustration of search and optimization algorithm 


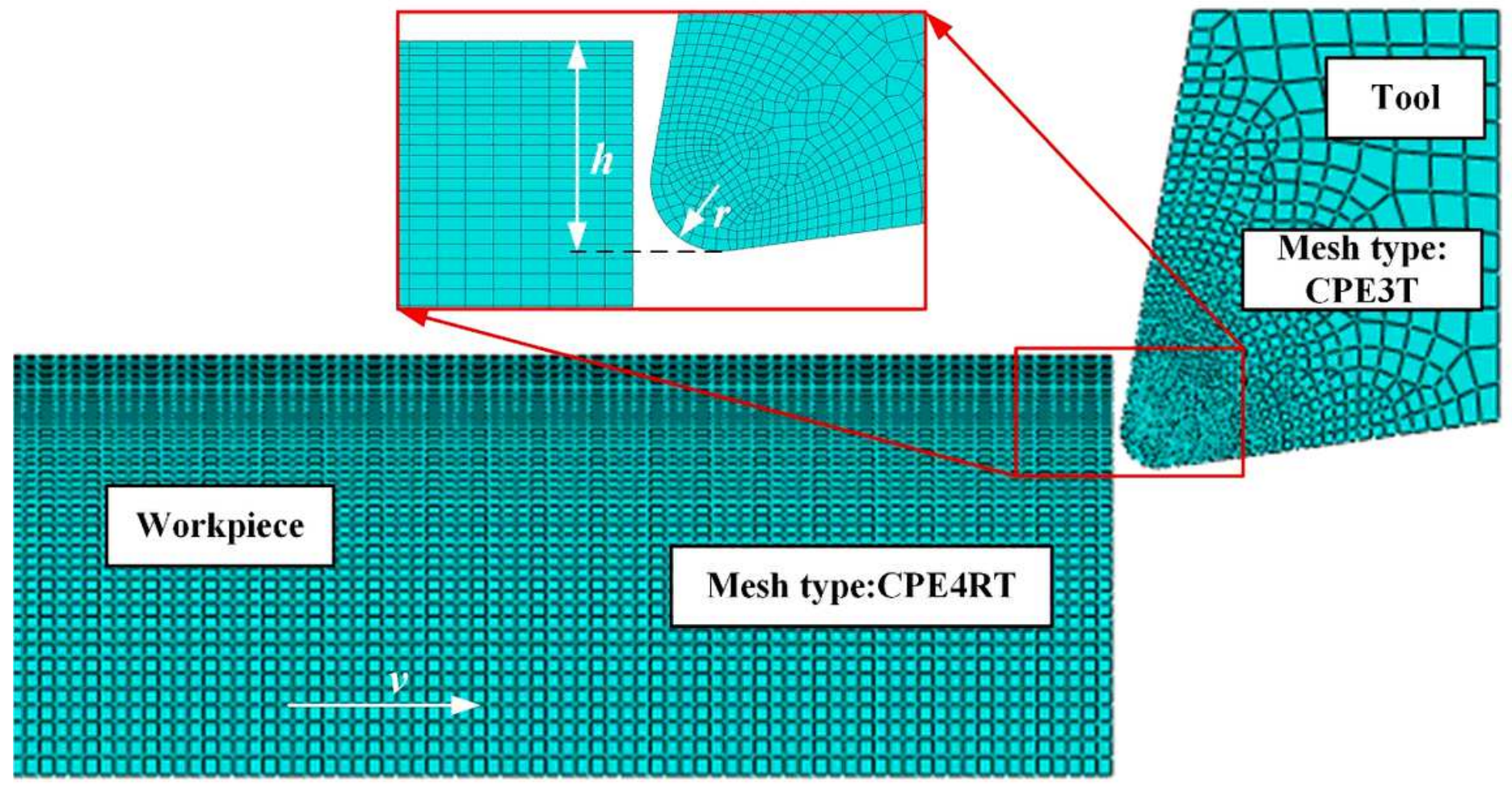

Figure 8

Geometrical modeling in finite element simulation

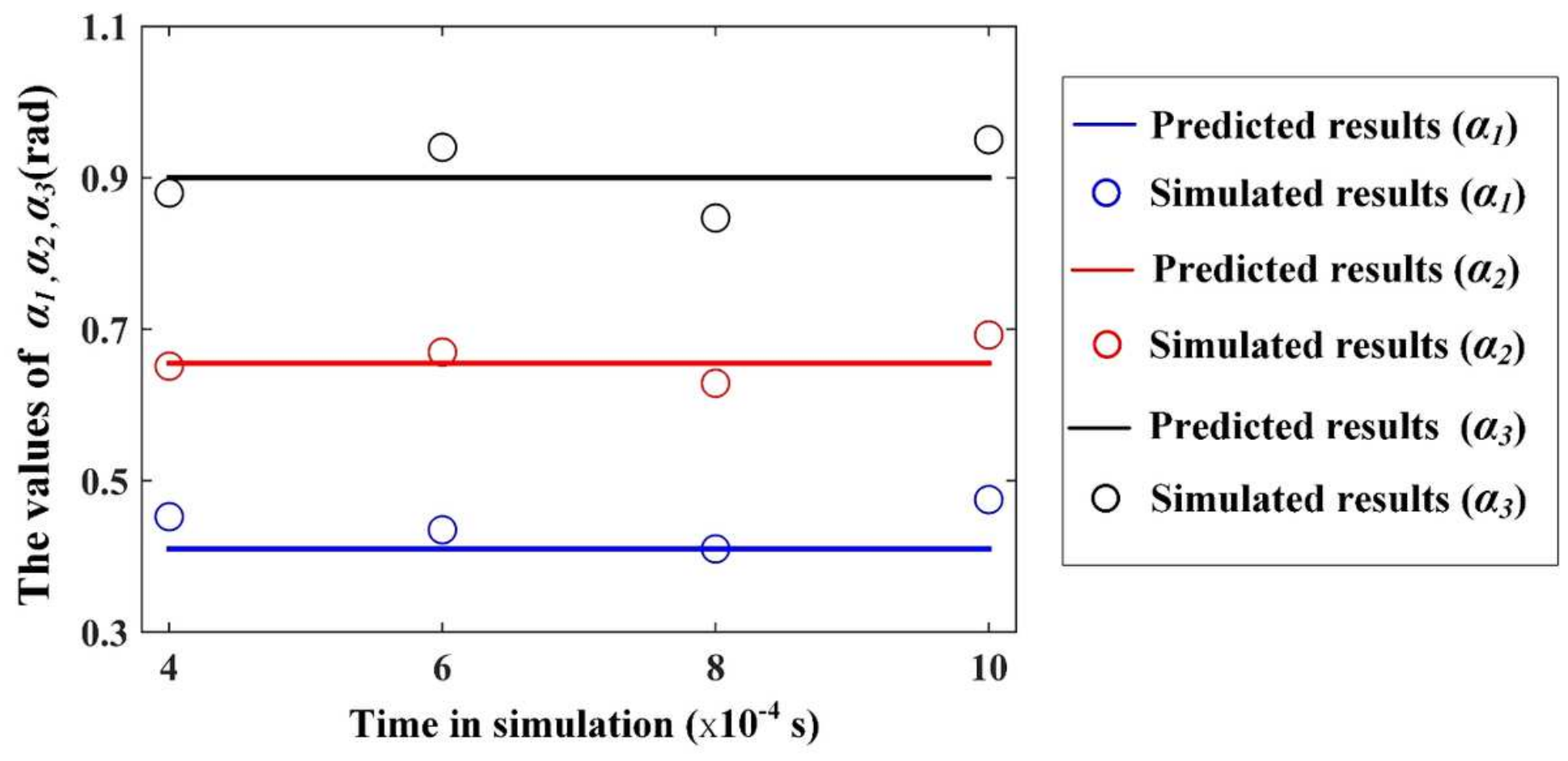

Figure 9 
The comparison of contact feature points between predicted results and simulated results $(r=0.04 \mathrm{~mm}$, $\mathrm{h}=0.1 \mathrm{~mm}$ )

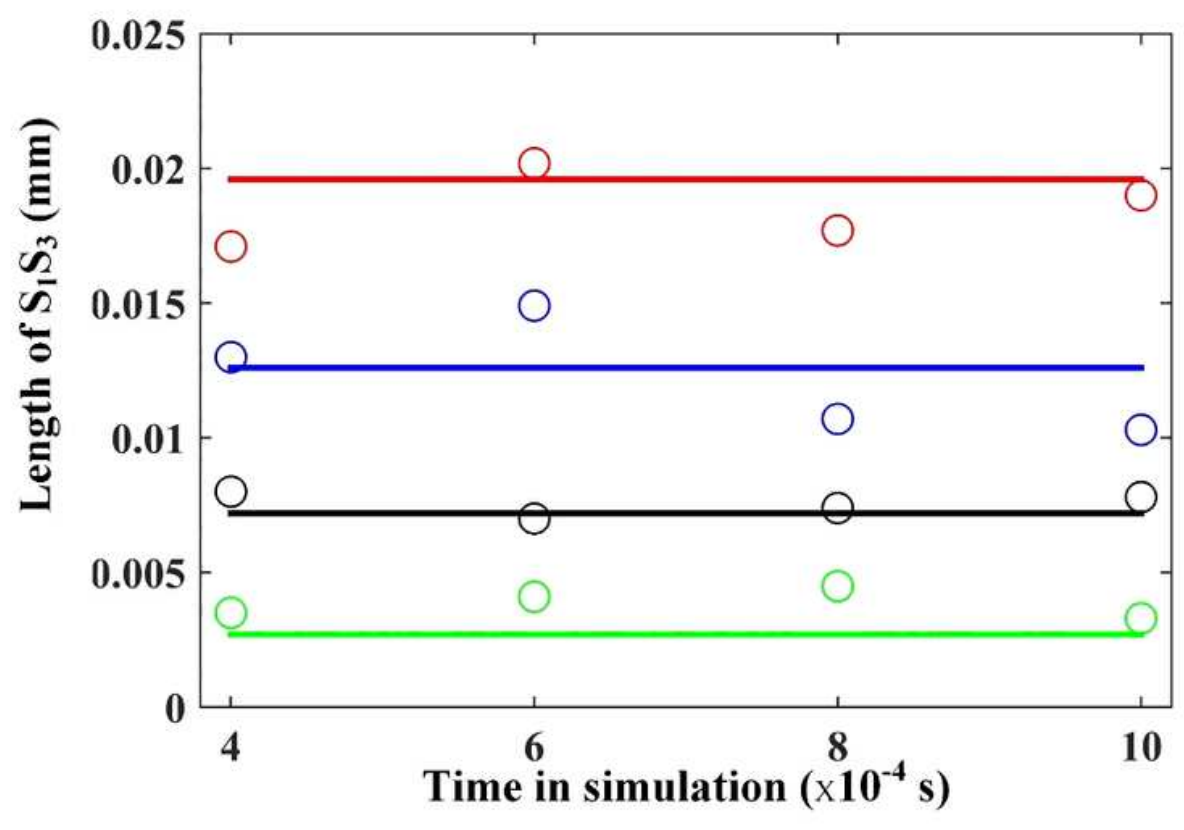

\begin{tabular}{|c|c|}
\hline & Predicted results $(r=0.01 \mathrm{~mm})$ \\
\hline 0 & Simulated results $(r=0.01 \mathrm{~mm})$ \\
\hline & Predicted results $(r=0.02 \mathrm{~mm})$ \\
\hline 0 & Simulated results $(r=0.02 \mathrm{~mm})$ \\
\hline & Predicted results $(r=0.03 \mathrm{~mm})$ \\
\hline $\mathrm{O}$ & Simulated results $(r=0.03 \mathrm{~mm})$ \\
\hline & Predicted results $(r=0.04 \mathrm{~mm})$ \\
\hline 0 & Simulated results $(r=0.04 \mathrm{~mm})$ \\
\hline
\end{tabular}

Figure 10

The comparison of radian distance between predicted results and simulated results 


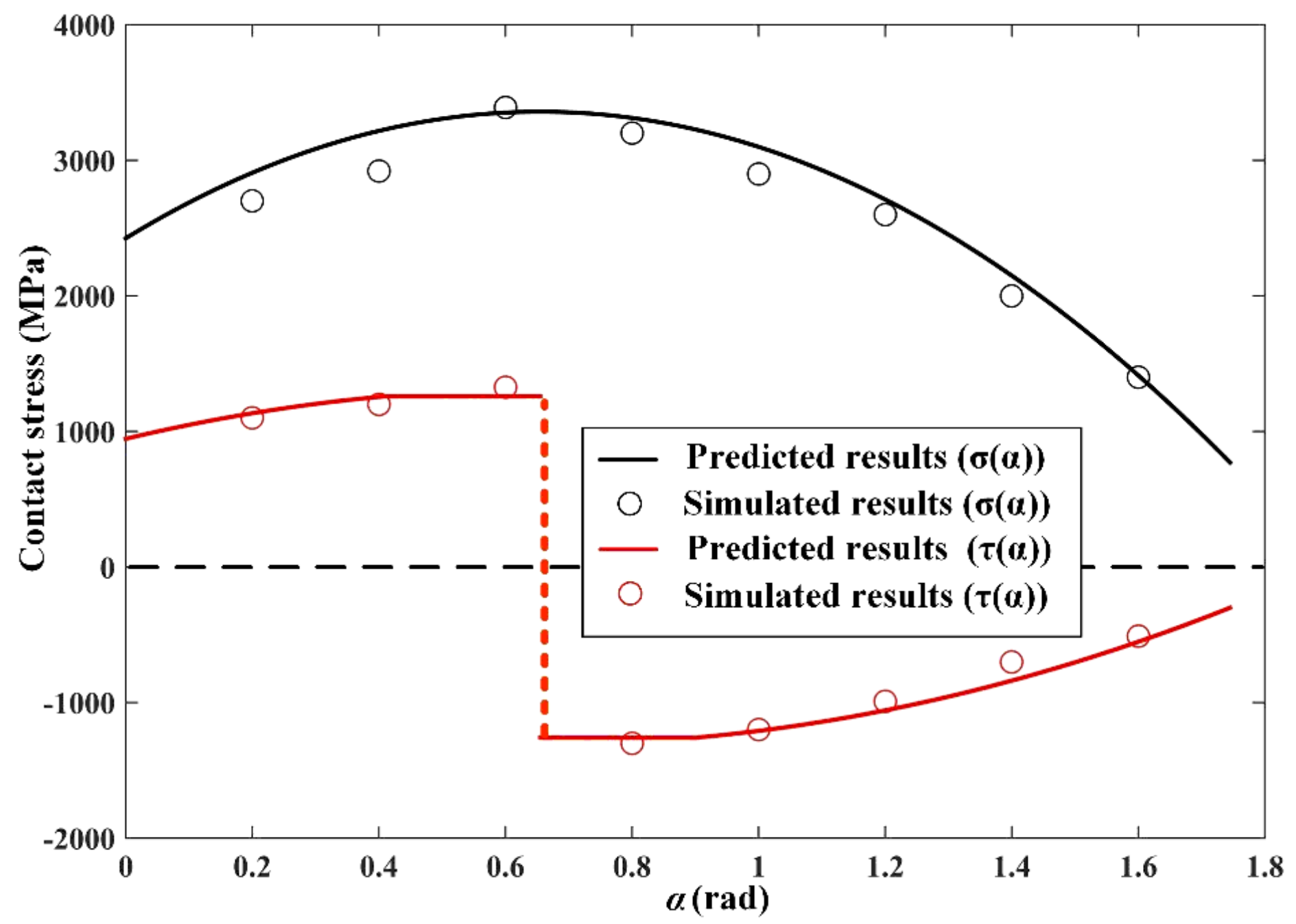

Figure 11

The contact stress comparison between predicted results and simulated results in round edge region $(\mathrm{r}=0.04, \mathrm{~h}=0.1)$
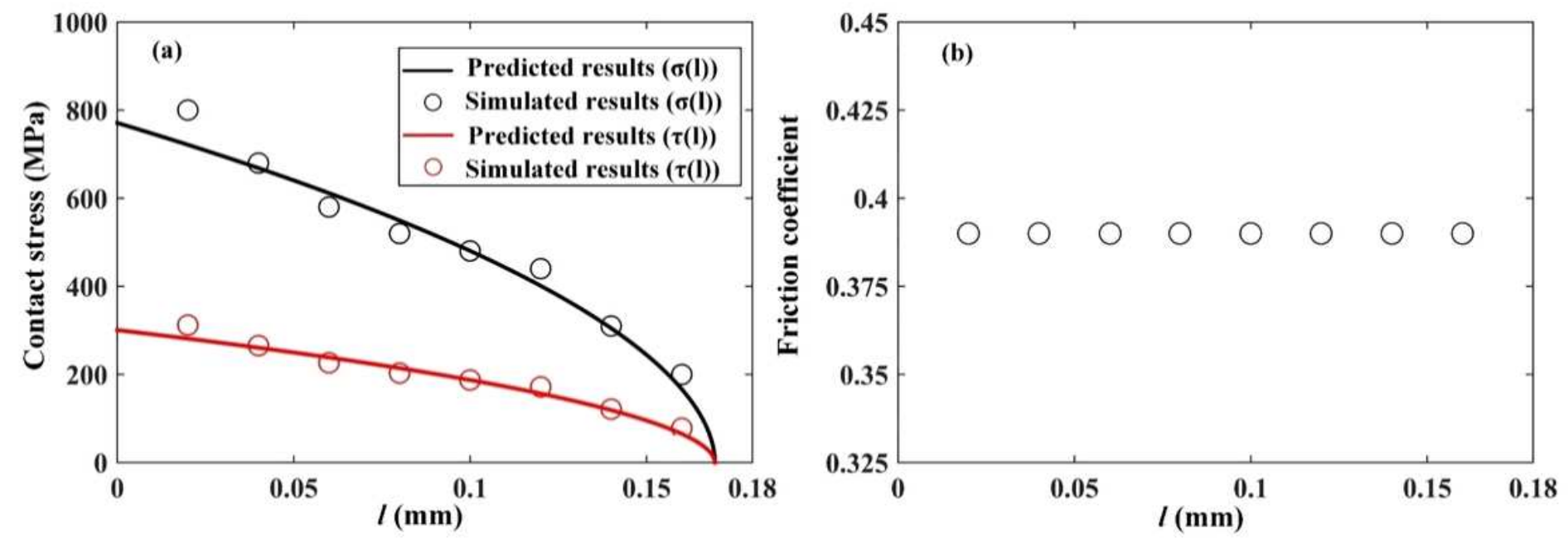

Figure 12 
Contact situation along the rake face with $\mathrm{r}=0.04$ and $\mathrm{h}=0.1$ (a. The co mparison between predicted results and simulated results, $b$. The frictional coefficient distribution)
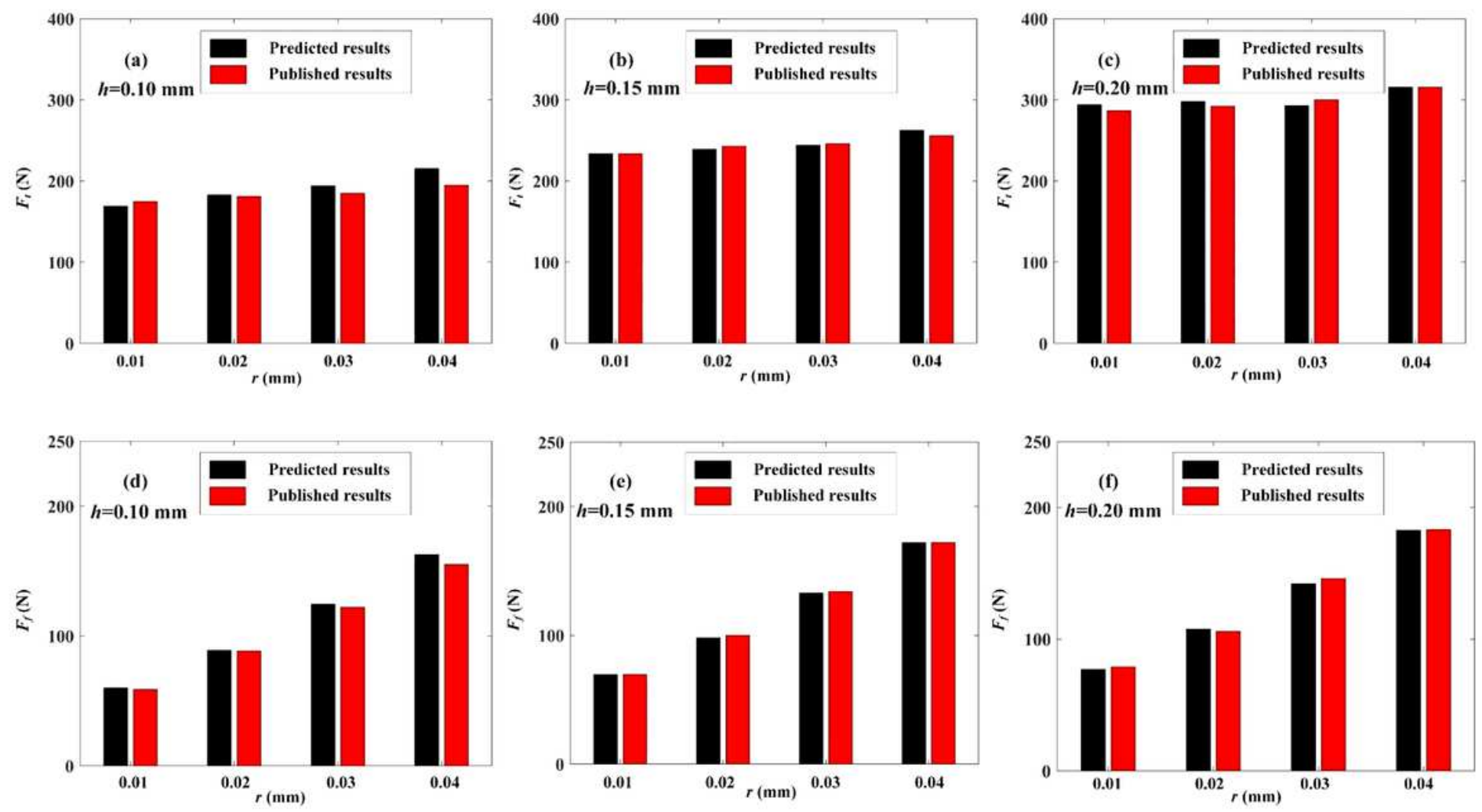

Figure 13

The force comparison between predicted results and published results 


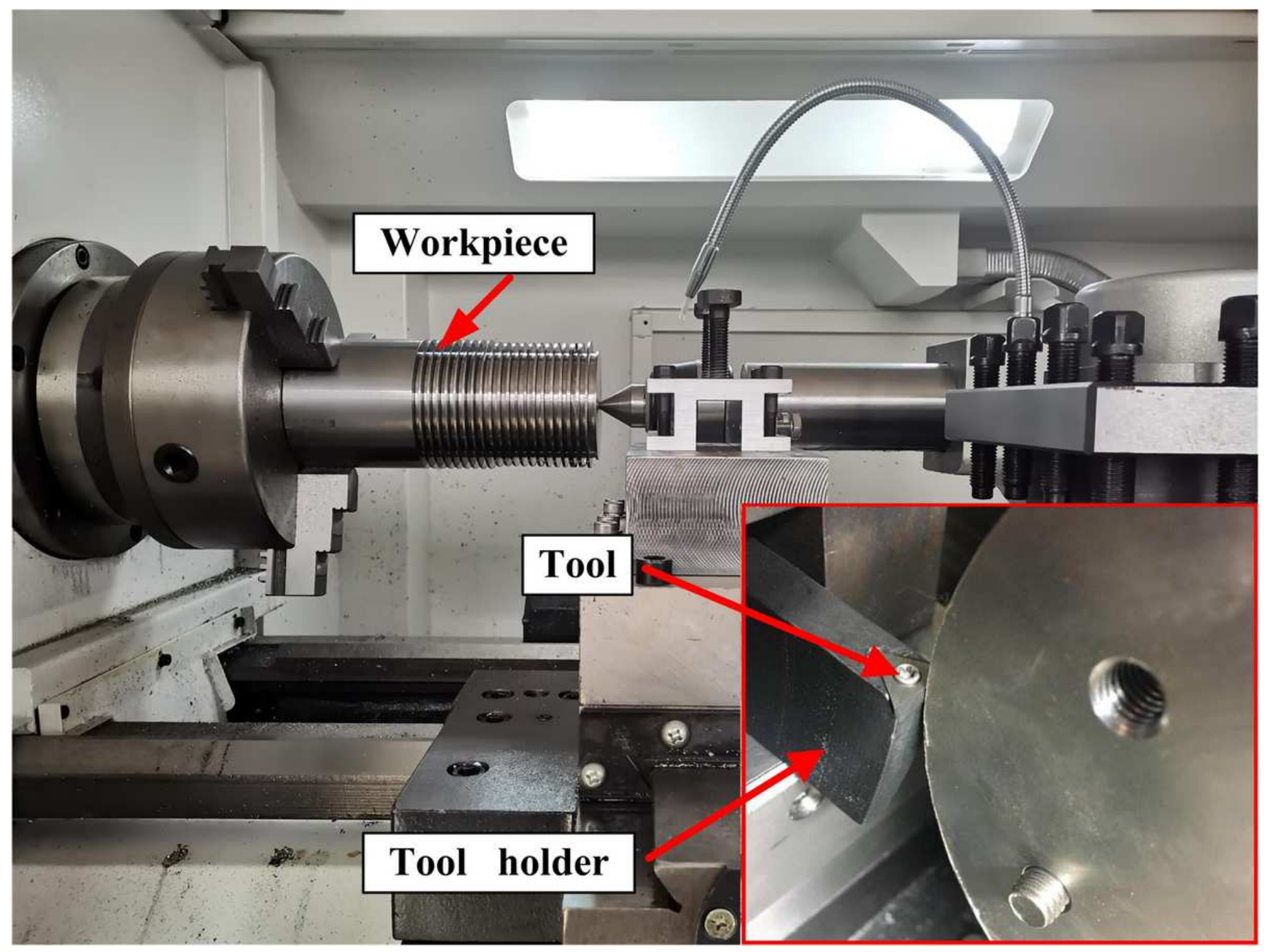

Figure 14

The experiment setup
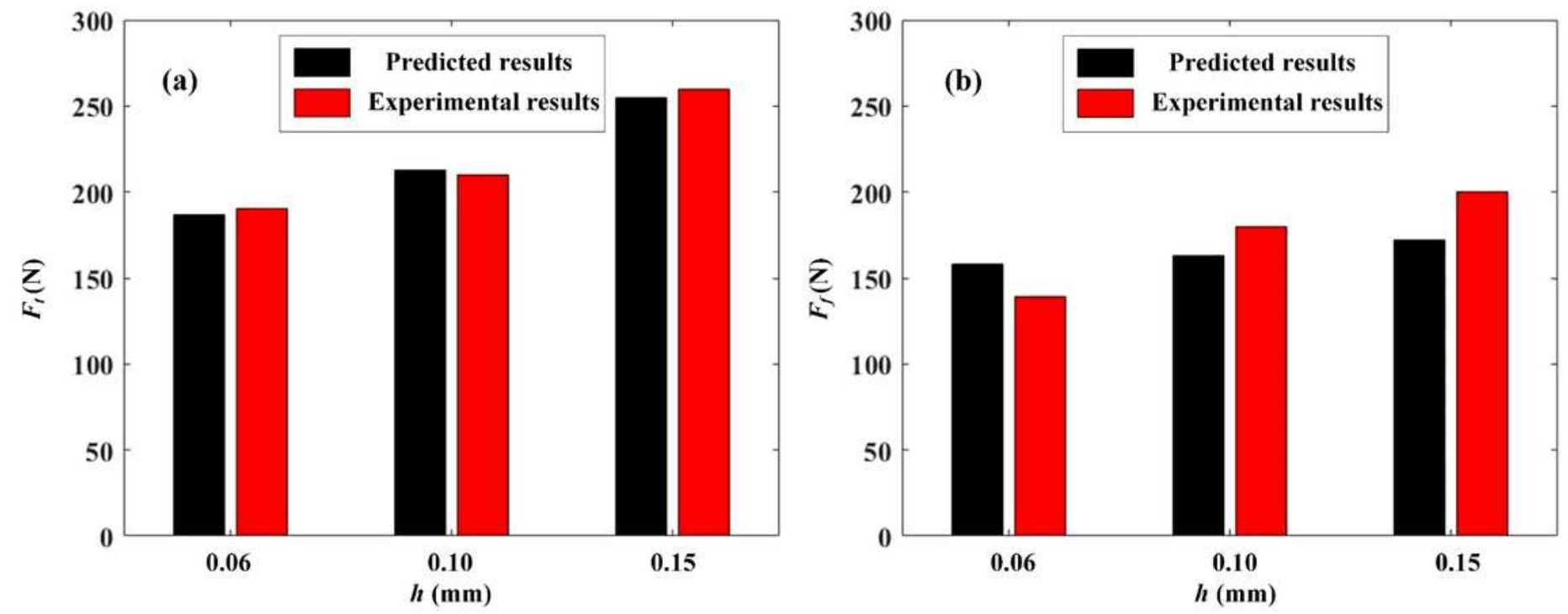


\section{Figure 15}

The forces comparison between predicted results and experimental results with $v=70 \mathrm{~m} / \mathrm{min}$ and $\mathrm{r}=0.04$ $\mathrm{mm}$ (a. Forces in cutting direction, b. Forces in feed direction)

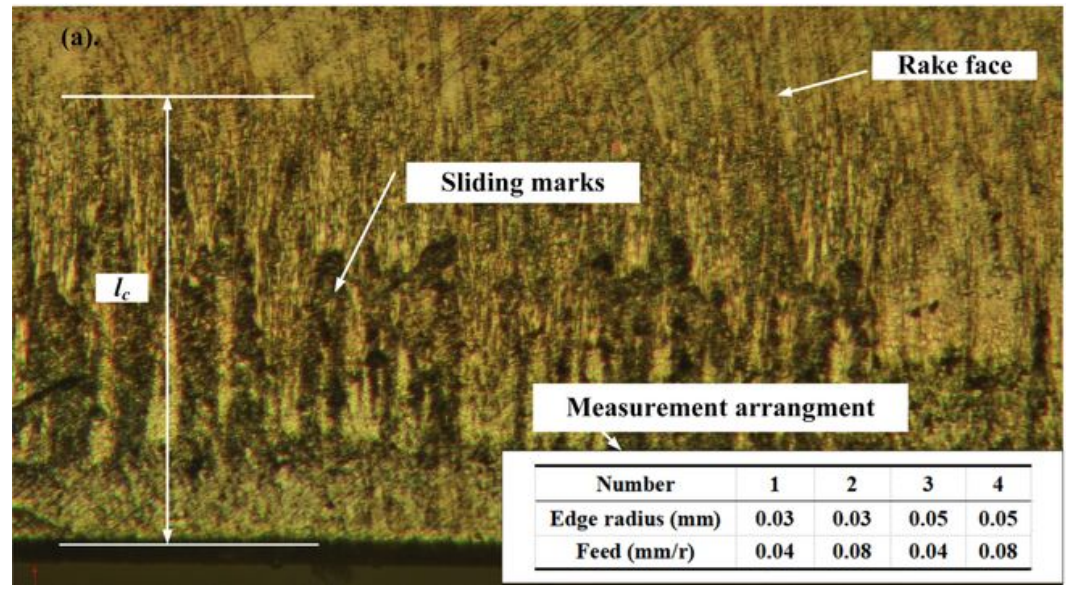

(b).

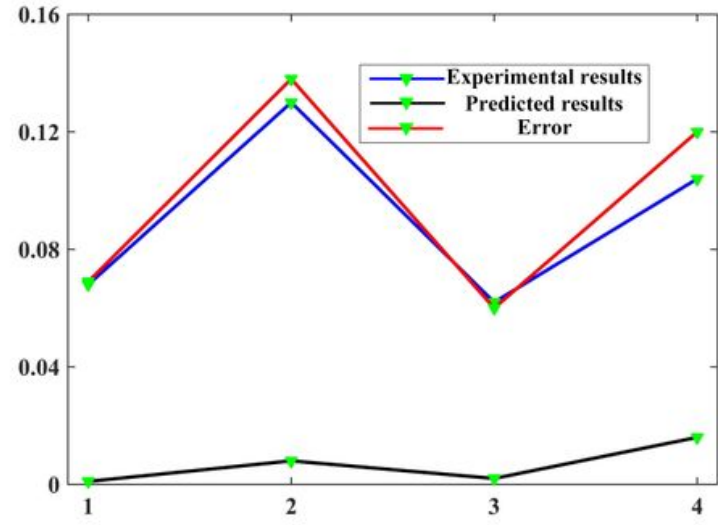

Figure 16

Contact length prediction in orthogonal cutting with carbide tool (a. The insert surface morphology in rake face; $b$. The contact length of experiment and prediction)

\begin{tabular}{|c|c|c|}
\hline \multicolumn{2}{|c|}{ Alicona setup } & Before cutting \\
\hline Cutting edge shape Leter cutting
\end{tabular}

Figure 17

The edge wear in orthogonal cutting 


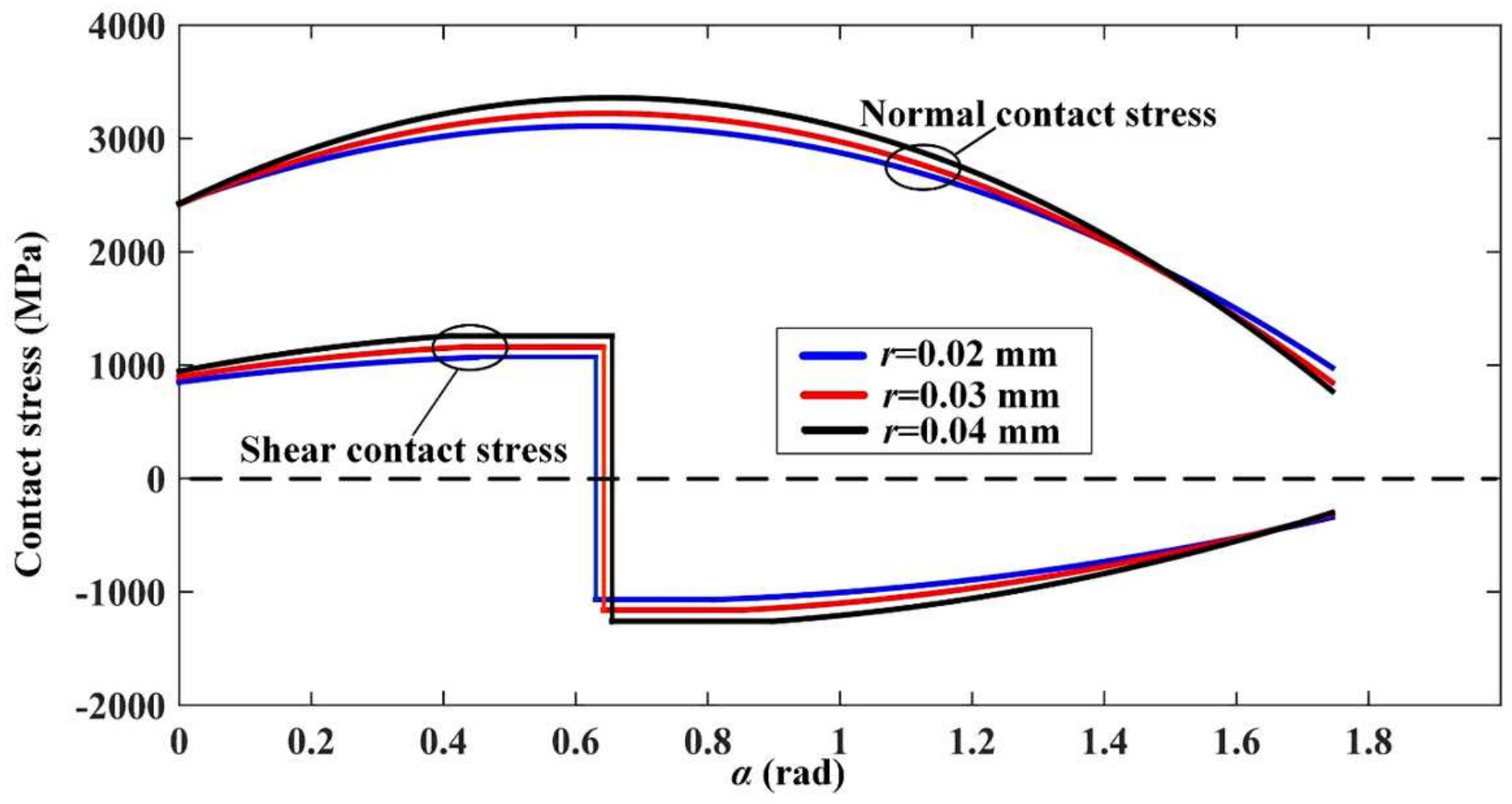

Figure 18

Contact stress distribution along the round edge with the proposed model (S0-S4) 


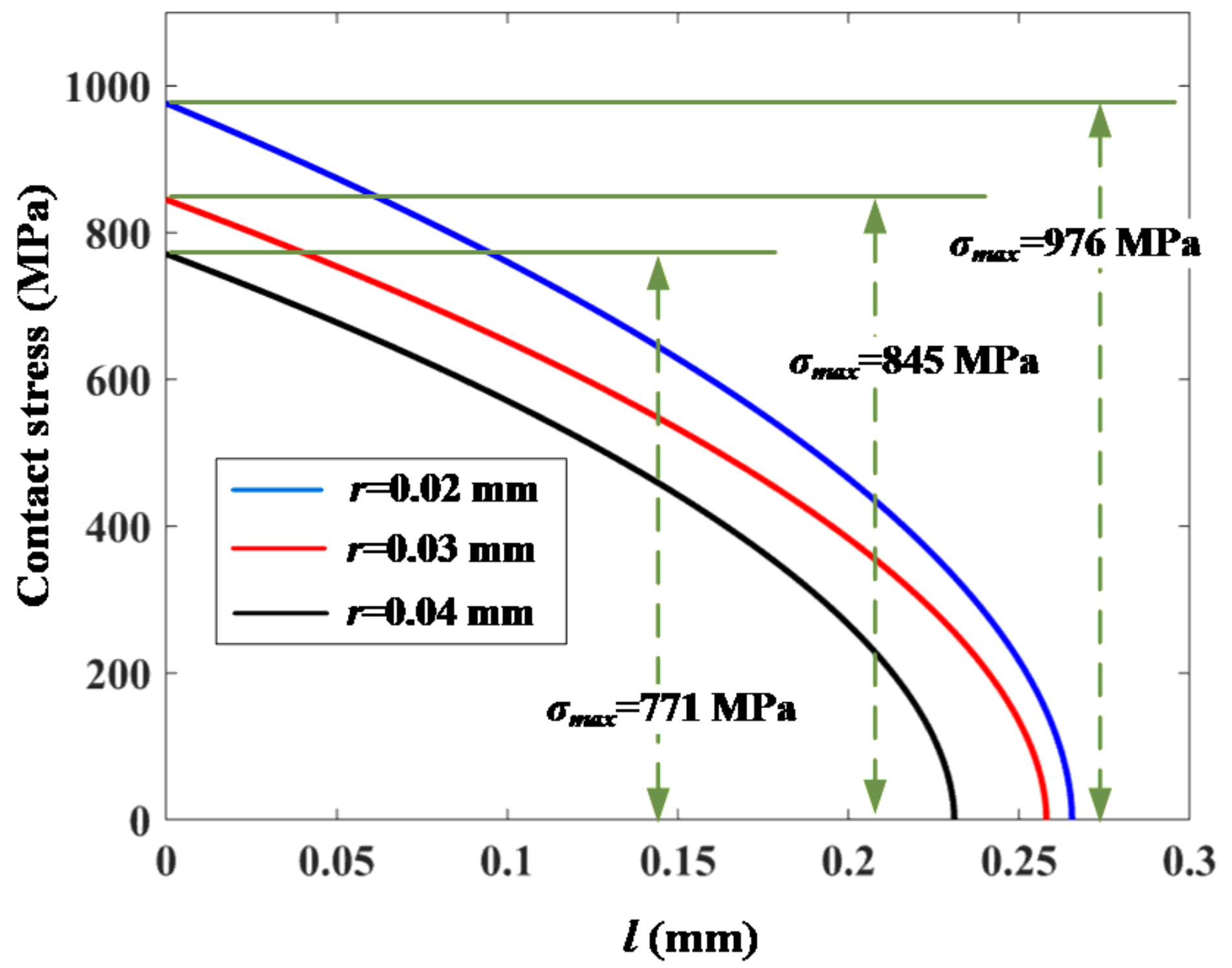

Figure 19

Normal contact stress along the rake face (S4-S5) $(h=0.10 \mathrm{~mm})$ 

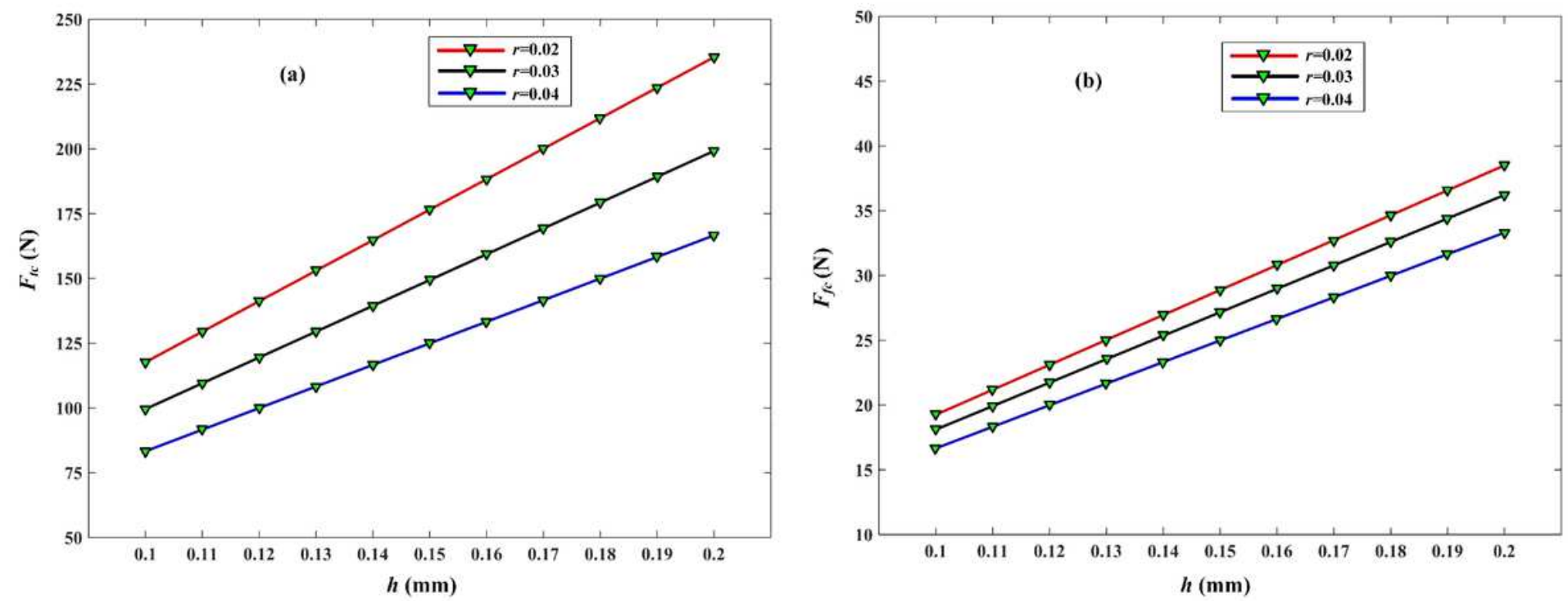

Figure 20

Contact force component along the rake face (a. Fc in cutting direction, b. Fc in feed direction)
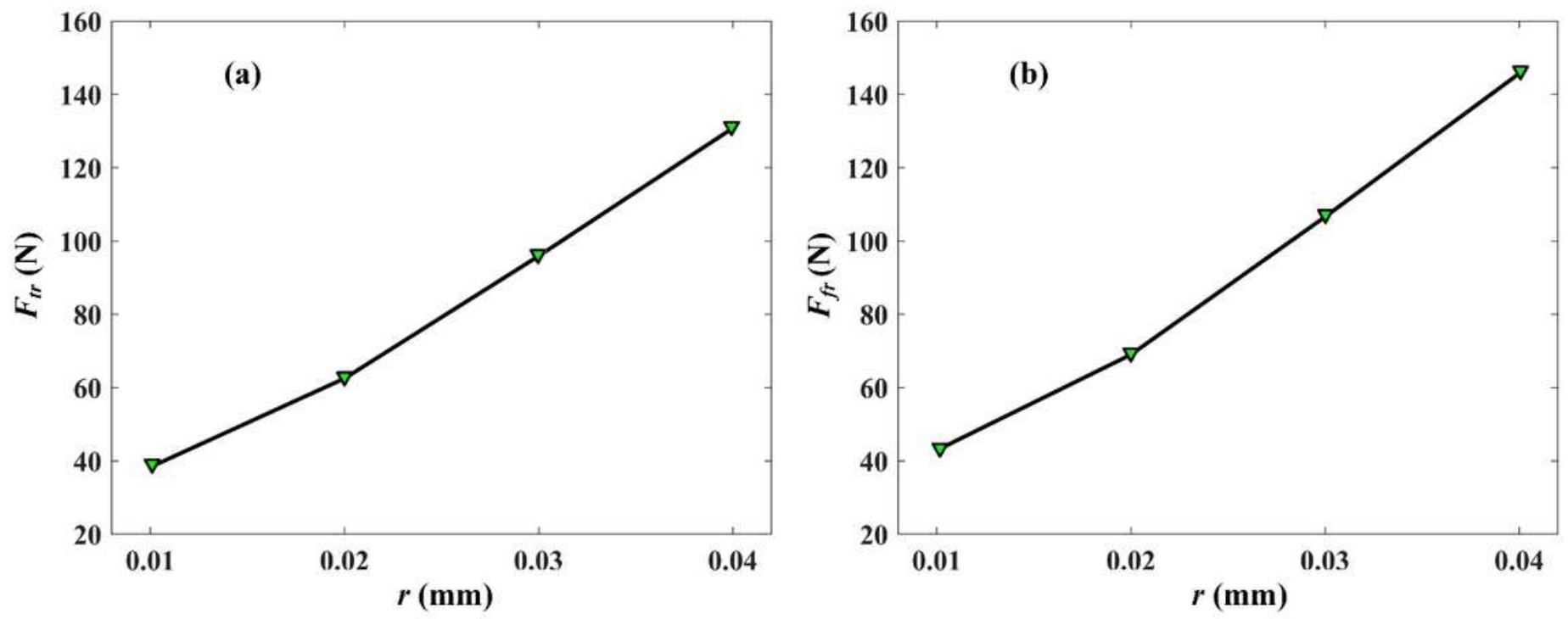

Figure 21

Contact force component along the round edge (a. Fr in cutting direction, b. Fr in feed direction) 